This article has been published in a revised form in Journal of Functional Programming https://doi.org/10.1017/s095679681600006x. This version is free to view and download for private research and study only. Not for re-distribution, re-sale or use in derivative works. $\odot$ Cambridge University Press 2016.

\title{
Transparent Fault Tolerance for Scalable Functional Computation
}

\author{
ROBERT STEWART \\ Mathematical \& Computer Sciences, Heriot-Watt University, Edinburgh, UK \\ PATRICK MAIER \& PHIL TRINDER \\ School of Computing Science, Glasgow, UK
}

\begin{abstract}
Reliability is set to become a major concern on emergent large-scale architectures. While there are many parallel languages, and indeed many parallel functional languages, very few address reliability. The notable exception is the widely emulated Erlang distributed actor model that provides explicit supervision and recovery of actors with isolated state.

We investigate scalable transparent fault tolerant functional computation with automatic supervision and recovery of tasks. We do so by developing $H d p H-R S$, a variant of the Haskell distributed parallel Haskell $(\mathrm{HdpH})$ DSL with Reliable Scheduling. Extending the distributed work stealing protocol of $\mathrm{HdpH}$ for task supervision and recovery is challenging. To eliminate elusive concurrency bugs, we validate the HdpH-RS work stealing protocol using the SPIN model checker.

HdpH-RS differs from the actor model in that its principal entities are tasks, i.e. independent stateless computations, rather than isolated stateful actors. Thanks to statelessness, fault recovery can be performed automatically and entirely hidden in the HdpH-RS runtime system. Statelessness is also key for proving a crucial property of the semantics of HdpH-RS: fault recovery does not change the result of the program, akin to deterministic parallelism.

HdpH-RS provides a simple distributed fork/join-style programming model, with minimal exposure of fault tolerance at the language level, and a library of higher level abstractions such as algorithmic skeletons. In fact, the HdpH-RS DSL is exactly the same as the HdpH DSL, hence users can opt in or out of fault tolerant execution without any refactoring.

Computations in HdpH-RS are always as reliable as the root node, no matter how many nodes and cores are actually used. We benchmark HdpH-RS on conventional clusters and an HPC platform: all benchmarks survive Chaos Monkey random fault injection; the system scales well e.g. up to 1400 cores on the HPC; reliability and recovery overheads are consistently low even at scale.
\end{abstract}

\section{Introduction}

We know from both data centres and High Performance Computing (HPC) that faults become frequent in large scale architectures, e.g. around $10^{5}$ cores (Barroso et al., 2013). With the exponential growth in the number of cores many expect servers with these numbers of cores to become commonplace in the near future. Hence the massively parallel computations on such architectures must be able to tolerate faults. For example there is already intense research to improve the fault tolerance of HPC software (Cappello, 2009), and cloud frameworks like Google MapReduce (Dean \& Ghemawat, 2008) and 
Hadoop (White, 2012) provide transparent fault tolerance for their restricted data retrieval computations.

There are many parallel functional languages e.g. (Marlow et al., 2009; Scholz, 2003), and many scale over distributed memory architectures to support massively parallel computations e.g. (Aljabri et al., 2014; Loogen et al., 2005). There are, however, very few fault tolerant parallel functional languages. The notable exception is the Erlang distributed actor model (Armstrong, 2010) that is widely emulated, e.g. by Cloud Haskell (Epstein et al., 2011) and the Akka library for Scala (Gupta, 2012). Erlang style fault tolerance provides mechanisms for the explicit supervision and recovery of stateful processes, or actors, and hence faults are observable.

We investigate exploiting the statelessness of functional languages to provide scalable transparent fault tolerance, in contrast to stateful actor models. If a copy of the closure representing a stateless computation is preserved it can be recovered by simply re-evaluating the closure. Conveniently functional languages like Haskell clearly distinguish stateless and stateful computations. There remain significant challenges to scaling parallel functional programs, e.g. they commonly employ complex data and control structures, and dynamically create tasks of irregular sizes.

The basis of our investigation is a variant of the Haskell Distributed Parallel Haskell DSL (HdpH), HdpH-RS that extends $\mathrm{HdpH}$ with reliable scheduling (RS). Both HdpH and HdpH-RS are designed for scalable distributed-memory parallelism, and employ work stealing to load balance dynamically-generated tasks of irregular sizes.

The paper makes the following research contributions.

Language design for transparent fault tolerance. The HdpH-RS implementations of the $\mathrm{HdpH}$ spawn and spawnAt primitives create and supervise idempotent tasks guaranteeing that they terminate providing the spawning location survives. Idempotent tasks have no side effects whose repetition can be observed, e.g. tasks that are stateless or perform idempotent database updates (Ramalingam \& Vaswani, 2013). By the transitivity of spawning the program terminates if the root node, where the program starts, survives 1 For ease of programming $\mathrm{HdpH}-\mathrm{RS}$ exploits the higher level $\mathrm{HdpH}$ programming abstractions, most significantly some 9 parallel skeletons that encapsulate common parallel programming patterns (Stewart \& Maier, 2013). Logically the programmer can switch from normal to fault tolerant execution simply by selecting the HdpH-RS implementation, i.e. without refactoring application code ${ }^{2}$

$\mathrm{HdpH}-\mathrm{RS}$ is designed to manage dynamic, irregular and idempotent task parallelism on large scale architectures like HPCs. It copes well with complex algorithms, coordination patterns, and data structures. There are many real-world applications with these characteristics, for example many combinatorial problems or computational algebra problems e.g. (Maier et al., 2014a). (Section3).

1 Erlang style automatic restarting of the root node could easily be provided, and would simply rerun the entire computation, typically up to a fixed failure frequency, e.g. 5 restarts/hour.

2 Section 6.4 outlines some minor pragmatic issues with using fault tolerance. 
An operational semantics for HdpH-RS that provides a concise and unambiguous model of scheduling in the absence and presence of failure. Provided that all tasks are idempotent, we show that the semantics makes failure unobservable: a program always computes the same result, no matter how many failed tasks had to be recovered (Sections 3.4 and 3.5.

The design and implementation of transparent fault tolerance via a distributed scheduler that implements the HdpH-RS spawn primitives, fault detection, and recovery. The reliable scheduler performs work stealing while tolerating the random loss of any or all nodes other than the root node. It replicates supervised tasks, tracks their location, and reinstates them if the task may have been lost. In the current implementation any tasks created by a lost task will also be recreated during the re-evaluation. The HdpH-RS implementation is publicly available (Stewart \& Maier, 2013) (Sections 4, 6).

A validation of the fault tolerant distributed scheduler with the SPIN model checker. The work stealing scheduling algorithm is abstracted in to a Promela model and is formally verified with the SPIN model checker (Holzmann, 2004). The model represents all failure combinations of non-root nodes that may occur in real architectures. The key reliability property shows that the variable representing a supervised task is eventually full despite node failures. The property is proven by an exhaustive search of 8.2 million states of the model's state space at a reachable depth of 124 transitions (Section 5).

An evaluation of the HdpH-RS transparent fault tolerance performance using four benchmarks on both conventional (Beowulf (Meredith et al., 2003)) clusters and the HECToR HPC (Edinburgh Parallel Computing Center (EPCC), 2008). All benchmarks execute without observable faults in the presence of Chaos Monkey (Hoff, 2010) random fault injection. We show that HdpH-RS scales well, e.g. achieving a speedup of 752 with explicit task placement and 333 with lazy work stealing when executing Summatory Liouville on 1400 HECToR cores. Supervision overheads are consistently low even at scale and recovery overheads are similarly low in the presence of frequent failure when lazy on-demand work stealing is used (Section 7). The dataset supporting this evaluation is available from an open access archive (Stewart et al., 2015).

Novelty This paper is the first comprehensive presentation of transparent fault tolerance in HdpH-RS, covering the design, implementation, semantics, validation, and evaluation. HdpH-RS was conceived as a key component of the SymGridPar2 scalable computational algebra framework. The HdpH-RS design is presented as part of the SymGridPar2 design in (Maier et al., 2014b), together with some very preliminary performance measurements. HdpH-RS is described alongside HdpH in (Maier et al., 2014c), but with only a few paragraphs dedicated to describing each of the implementation, validation, and evaluation.

\section{Related Work}

\subsection{Faults and Reliability}

A fault is a characteristic of hardware or software that can lead to a system error. An error can lead to an erroneous system state giving a system behaviour that is unexpected 
by system users. Faults may be due to a range of factors, e.g. incorrect software, or the capacity issues like insufficient memory or persistent storage.

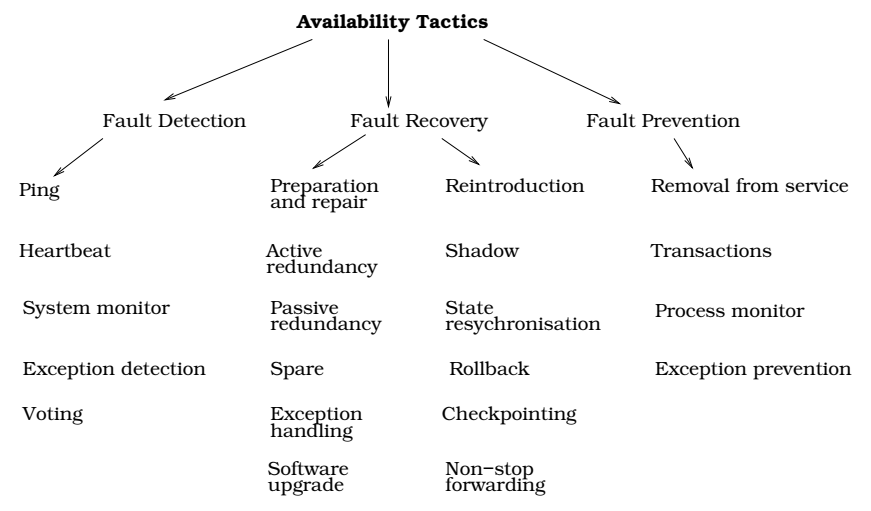

Fig. 1: Availability Tactics from (Scott \& Kazman, 2009)

Tactics for fault tolerance detection, recovery and prevention are shown in Figure 1 Monitors are components that can monitor many parts of a system, such as processors, nodes, and network congestion. They may use heartbeat or ping-pong protocols to monitor remote components in distributed systems. In message passing distributed systems, timestamps can be used to detect or re-order incorrect event sequences. Most existing work in fault tolerance for HPC systems is based on checkpointing or rollback recovery. Checkpointing methods (Chandy \& Lamport, 1985) are based on periodically saving a global or semi-global state to stable storage. Global checkpointing does not scale, and though asynchronous checkpointing approaches have potential to scale on larger systems, they encounter difficult challenges such as rollback propagation, domino effects, and loss of integrity through incorrect rollback in dependency graphs (Elnozahy et al., 2002). Algorithmic level fault tolerance is an alternative high level fault tolerance approach. Examples include leader election algorithms, consensus through voting and quorums, or simply ignore failures by smoothing over missing results with probabilistic accuracy bounds.

\subsection{Reliability of Large Scale Systems}

The success of future HPC architectures will depend on the ability to provide reliability and availability at scale (Schroeder \& Gibson, 2007). As HPC systems continue to increase in scale, their mean time between failure decreases. The current practise for fault tolerance in HPC systems is to use very reliable hardware, e.g. processors and interconnects, and to use checkpointing and rollback. With the increasing error rates and increasing aggregate memory exceeding IO capabilities, checkpointing is fast becoming unusable (Litvinova et al., 2010).

Parallel functional computations are typically very different from most HPC computations that perform relatively regular iterations over arrays of floating points. In contrast $\mathrm{HdpH}$ and other functional computations employ complex data and control structures, use arrays and floating points sparingly, and dynamically create tasks of irregular sizes. Rather than checkpointing, HdpH-RS uses supervision and re-evaluation for fault tolerance. 
Big Data frameworks like Google MapReduce (Dean \& Ghemawat, 2008), and Hadoop (White, 2012) operate at scale on commodity hardware and hence provide fault tolerance. They provide automatic and largely transparent fault tolerance. Queries are idempotent, the data is replicated in a distributed file store, e.g. by the Hadoop HDFS (White, 2012), the failure of a sub query is detected and it is recomputed. Such frameworks perform a restricted form of data retrieval computation. HdpH-RS, and other parallel functional languages, are more commonly used for large compute-bound tasks, i.e. Big Computation and not Big Data.

HdpH-RS differs from Hadoop in a number of ways. Where a Hadoop master node supervises the health of all slave nodes, supervision is distributed in $\mathrm{HdpH}-\mathrm{RS}$, and all $\mathrm{HdpH}$ RS nodes are capable of detecting remote node failures. In Hadoop, failure detection and task replication is centralised. The output of map tasks are stored to disk locally in Hadoop. In the presence of failure, completed map tasks are redundantly re-scheduled, due to the loss of their results. This is in contrast to HdpH-RS, where the resulting values of evaluating task expressions is transmitted with rput as soon as they are calculated. Failure detection latency in Hadoop is 10 minutes by default in order to tolerate non-responsiveness and network congestion (Dinu \& Ng, 2011). In contrast, the failure detection latency in $\mathrm{HdpH}$ RS is a maximum of 5 seconds by default, and can be modified by the user.

\subsection{Fault Tolerant Distributed Languages}

Erlang is a distributed actor based functional language that is increasingly popular for developing reliable scalable systems, many with soft real-time requirements. The Erlang approach to failures relies on actors having isolated state, and hence they can 'let it crash' and rely on another process to correct the error (Armstrong, 2010). One Erlang process can monitor another Erlang process, which is notified if the monitored process dies. The Erlang reliability model is widely emulated, e.g. by and the Akka library for Scala (Gupta, 2012) and by Cloud Haskell (Epstein et al., 2011).

The Cloud Haskell and HdpH-RS designs and implementations are closely related. Both languages are implemented entirely in Haskell with GHC extensions and inherit the language features of Haskell, including purity, types, and monads, as well as the multiparadigm concurrency models in Haskell. Both provide mechanisms for serialising function closures, enabling higher order functions to be used in distributed computing environments. Both are de-coupled into multiple layers, separating the process layer, transport layer, and transport implementations. The software architecture, illustrated in Figure 2 is designed to encourage additional middlewares other than Cloud Haskell for distributed computing, and for alternative network layer implementations other than TCP. Cloud Haskell and HdpH-RS share the TCP network layer.

HdpH-RS reliability differs from Erlang-style reliability in a number of ways, and for concreteness we compare with distributed Erlang. HdpH-RS provides dynamic load management where an idle node may steal a task. In contrast distributed Erlang spawns a process to a named node (host), and thereafter it cannot migrate. This makes Erlang less suitable than HdpH-RS for computations with irregular parallelism. HdpH-RS recovery in effect automates Erlang OTP supervision behaviours and hence user does not need to handle failures excplicity as in Erlang. Where HdpH-RS is designed to recover only 


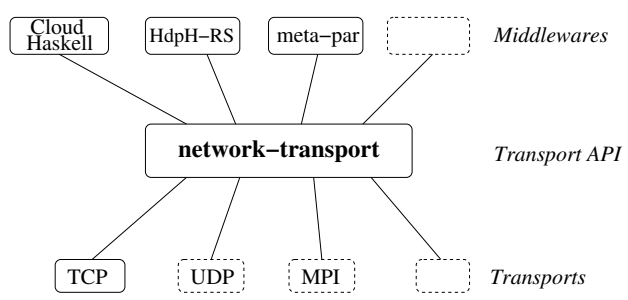

Fig. 2: Distributed Haskell Software Layers

idempotent tasks transparently, i.e. without making faults observable, the recovery of a stateful process in Erlang may make the faults observable. Such a model can provide better availability as nodes (Erlang VMs) can be monitored and restarted from the operating system. In contrast to HdpH-RS and Cloud Haskell Erlang is dynamically typed, and hence programming errors may be detected only at runtime.

\section{Design}

\subsection{HdpH-RS Language}

Haskell Distributed Parallel Haskell (HdpH) and HdpH-RS are shallowly embedded Haskell DSLs that support high-level explicit and semi-explicit parallelism. HdpH-RS is scalable as it distributes computations across a network of multicore nodes. It is portable, being implemented entirely in Haskell (with GHC extensions) rather than relying on bespoke low-level runtime systems like Glasgow parallel Haskell $(\mathrm{GpH})$ (Trinder et al., 1996) or Eden (Loogen et al., 2005).

HdpH-RS provides high-level semi-explicit parallelism with implicit and explicit task placement and dynamic load management. Implicit placement frees the programmer from controlling work distribution and load management. Instead, idle nodes steal work from busy nodes automatically, thereby maximising utilisation when there is enough work to be stolen at the expense of deterministic execution. HdpH-RS provides low cost automatic fault tolerance using Erlang-style supervision and recovery of location-invariant computations.

HdpH-RS extends the Par monad DSL (Marlow et al., 2011) for shared-memory parallelism to distributed memory, and Listing 1 lists the HdpH API. Like (Marlow et al., 2011), HdpH focuses on task parallelism. In distributed memory, this requires serialisation of Par computations and results so they can be sent over the network. The Par type constructor is a monad for encapsulating a parallel computation. To communicate the results of computation (and to block waiting for their availability), threads employ IVars as futures (Halstead Jr., 1985), which are essentially mutable variables that are writable exactly once.

The spawn and spawnAt primitives immediately return a future of type IVar (Closure t). The actual result can be read by calling get, blocking until the result is available. Note that a future is not serialisable, hence cannot be captured by explicit closures. As a result the future can only be read on the hosting node, i.e. the node it was created on. Details of explicit closures in HdpH-RS are in (Maier et al., 2014b). 


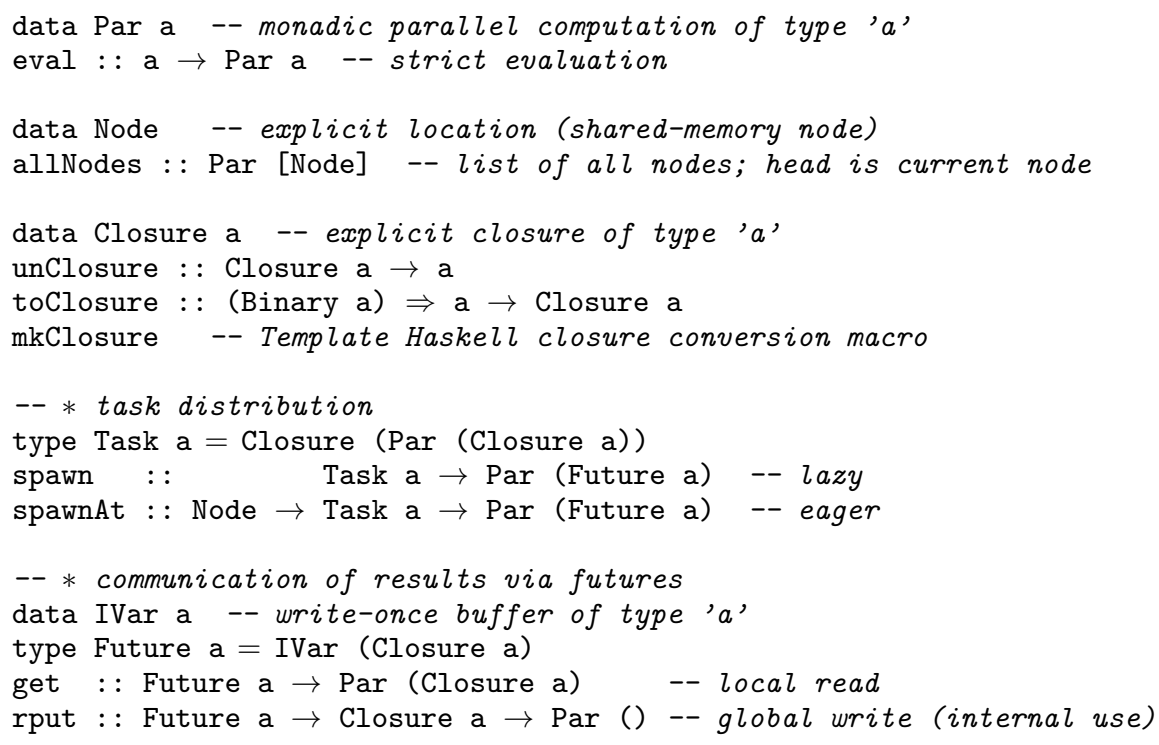

\section{Listing 1: HdpH-RS Primitives}

The example in Listing 2 illustrates the use of the $\mathrm{HdpH}$ primitives to sum the Liouville function (Borwein et al., 2008) from 1 to $n$ in parallel. The code shows how to construct a list of tasks with the mkClosure macro, how to generate parallelism by spawning the tasks, how to retrieve the results closures, and how to unwrap them and return the final sum. Note how the actual parallel computation, the function liouville, applies the toClosure primitive to create a result closure containing a fully evaluated Integer.

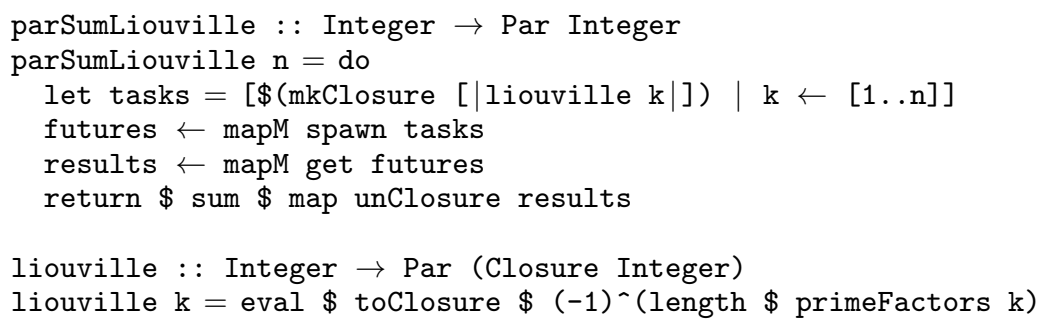

Listing 2: Summatory Liouville with HdpH-RS

\subsection{Algorithmic Skeletons}

HdpH-RS provides algorithmic skeletons (Cole, 1988) that abstract over the DSL primitives to provide high level fault tolerant parallelism. Algorithmic skeletons abstract communication, interaction, and commonly-used patterns of parallel computation e.g. divideand-conquer, map/reduce, parallel map and parallel buffer variants. Programmers that use 
these skeletons do not create tasks with spawn, spawnAt or call get on IVars directly. Instead, the skeleton implementations handle task creation and placement and synchronisation using IVars.

There are two versions of each algorithmic skeleton, one that uses eager scheduling and another that uses lazy scheduling. Lazy skeletons are implemented with combinations of spawn and get, and rely on the work stealing scheduler to load balance tasks. Eager skeletons are implemented with combinations of spawnAt and get, and push tasks to nodes as soon as they are created. Node selection with spawnAt varies between skeletons. Parallel map skeletons e.g. pushMap and pushMapSliced select nodes in a round-robin fashion. Nested skeletons e.g. pushDivideAndConquer (Stewart, 2013b) use random node selection. We explore the performance implications of fault tolerance and lazy/eager scheduling in Section7

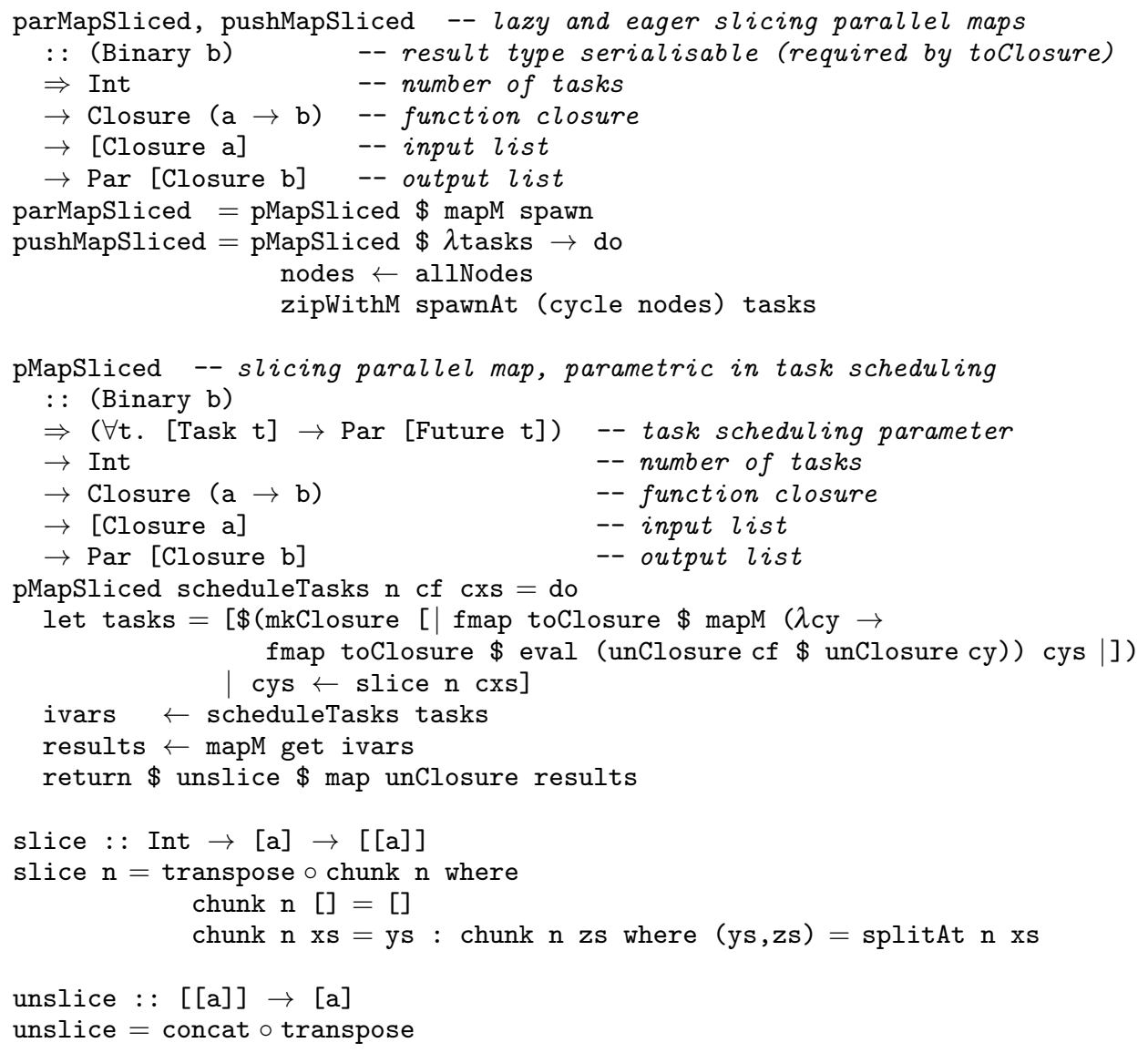

Listing 3: Two versions of a HdpH-RS Skeleton

Two versions of an HdpH-RS parallel map skeleton are shown in Listing 3 Both divide the input list into a number of slices and evaluate each slice in parallel. For example, 
dividing the list $\left[e_{1}, \ldots, e_{5}\right]$ into three slices yields a list $\left[\left[e_{1}, e_{4}\right],\left[e_{2}, e_{5}\right],\left[e_{3}\right]\right]$. The skeletons create three parallel tasks, distributed lazily with parMapSliced and eagerly with pushMapSliced. The two skeletons actually differ only in task scheduling; task creation and gathering of results, including slicing and unslicing of lists of closures, is uniformly handled by the underlying parametric skeleton pMapSliced. The full list of HdpH-RS skeletons are in (Stewart, 2013b) and their implementations are online (Stewart \& Maier, 2013).

\subsection{Applicability}

HdpH-RS is designed to manage dynamic, irregular and idempotent task parallelism on large scale architectures like large clusters or HPCs. Section 7 demonstrates that HdpHRS delivers good performance for applications with complex algorithms, coordination patterns, and data structures. As HdpH-RS retains backup closures of supervised tasks, its performance is predicated on a small closure footprint: either there are few closures, or they are small, or terminate quickly. Thus, HdpH-RS offers a trade-off between fault tolerance and memory use. There are many real-world applications with these characteristics, for example many combinatorial problems or computational algebra problems e.g. (Maier et al., 2014a).

On the other hand HdpH-RS is not appropriate for certain classes of application. In particular we do not target traditional HPC workloads, i.e. regular computations over vectors of floating points. These have little need for the dynamic load management and rich data structures provided by $\mathrm{HdpH}-\mathrm{RS}$, and need highly optimised floating point and vector capabilities. Similarly, for good performance task execution time must greatly outweigh communication time, which is largely determined by the size of the closure transmitted. Hence Big Data workloads with large memory footprints are not well suited. Finally HdpHRS would not facilitate the development of applications that rely on reliable distributed data structures, like replicated distributed hash tables, as these are not yet provided.

\subsection{Operational Semantics}

This section presents an operational semantics for HdpH-RS in the style of (Marlow et al., 2011), focusing on fault recovery. Figure 3 introduces the syntax of terms and values. IVars are represented as names which can be bound by name restriction $v$; the set of names occuring free in $X$ (where $X$ may be of any of the syntactic categories in Figure 3) is denoted by $f n(X)$.

The term language of Figure 3 is essentially the same as the embedded DSL presented in Section 3.1. except that it ignores explicit closures, i.e. assumes that all terms are implicitly serialisable. We assume that terms that may be serialised have no free IVars, more precisely, we restrict the last argument of spawn, spawnAt and rput to terms $M$ such that $f n(M)=\emptyset$. This is justified as in the language design (Section 3.1) these arguments are explicit closures that cannot capture free IVars.

For the purposes of the DSL semantics the host language is a standard lambda calculus with fixed points and some data constructors for nodes, integers and lists (omitted to save space). We assume a big-step operational semantics for the host language, and write $M \Downarrow V$ 


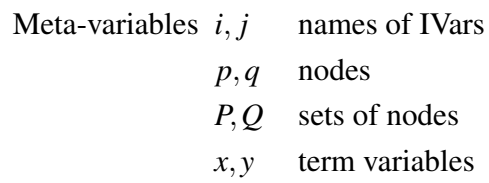

Values $V::=()|i| p\left|x M_{1} \ldots M_{n}\right| \lambda x . M \mid \mathbf{f i x} M$

$|M \gg>=N| \operatorname{return} M \mid$ eval $M \mid$ allNodes $|\operatorname{spawn} M| \operatorname{spawnAt} p M|\operatorname{get} i| \operatorname{rput} i M$

Terms $\left.L, M, N::=V|M N|(\rangle^{\prime}=\right) \mid$ return | eval | spawn | spawnAt | get | rput

States $R, S, T::=S \mid T \quad$ parallel composition

| vi.S name restriction

$\mid\langle M\rangle_{p} \quad$ thread on node $p$, executing $M$

$\mid\langle\langle M\rangle\rangle_{p} \quad$ spark on node $p$, to execute $M$

| $i\{M\}_{p} \quad$ full IVar $i$ on node $p$, holding $M$

$\mid i\left\{\langle M\rangle_{q}\right\}_{p} \quad$ empty IVar $i$ on node $p$, supervising thread $\langle M\rangle_{q}$

$\mid \quad i\left\{\langle\langle M\rangle\rangle_{Q}\right\}_{p}$ empty IVar $i$ on node $p$, supervising spark $\langle\langle M\rangle\rangle_{q}$ for some $q \in Q$

$\mid i\{\perp\}_{p} \quad$ zombie IVar $i$ on node $p$

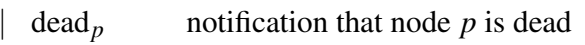

Evaluation contexts $\mathscr{E}::=[\cdot] \mid \mathscr{E}>>=M$

Fig. 3: Syntax of HdpH-RS terms, values and states.

to mean that there is a derivation proving that term $M$ evaluates to value $V$. The definition of the host language semantics is entirely standard and omitted (Peyton Jones, 2002). Note that the syntax of values in Figure 3 implies that the DSL primitives are strict in arguments of type Node and IVar.

The operational semantics is a small-step reduction semantics $\longrightarrow$ on the states defined in Figure 3 A state is built from atomic states by parallel composition and name restriction. Each atomic state has a location, a node indicated by the subscript $p$. The special atomic

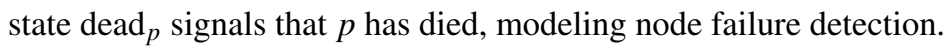

An atomic state of the form $\langle M\rangle_{p}$ or $\langle\langle M\rangle\rangle_{p}$, where $M$ is a computation of type Par (), denotes a thread or spark, respectively. A thread is a currently executing task and is tied to its current node $p$; a spark is a task that does not yet execute and may migrate from $p$ to any other node $q$. An atomic state of the form $i\{?\}_{p}$ denotes an IVar named $i$; the place holder “?” signals that we don't care whether $i$ is full, empty, or a zombie. To enable fault recovery, empty IVars $i\left\{\langle M\rangle_{q}\right\}_{p}$ and $j\left\{\langle\langle N\rangle\rangle_{Q}\right\}_{p}$ supervise the thread $\langle M\rangle_{q}$ resp. spark $\langle\langle N\rangle\rangle_{q}$ that is supposed to fill them, maintaining knowledge of their location. In case of a non-migratable thread that knowledge is the exact node $q$ where it was placed by the scheduler. In case of a spark, however, the superving IVar $j$ may not know the actual node due to migration, hence the spark is annotated with a set of nodes $Q$ over-approximating its true location. Figure 4 asserts the usual structural congruence properties of parallel composition and name restriction, and the usual structural transitions propagating reduction under parallel composition and name restriction. 


$$
\begin{array}{rlrl}
S \mid T & \equiv T \mid S & \text { vi.vj.S } & \equiv \text { vj.vi.S } \\
R|(S \mid T) \equiv(R \mid S)| T & \text { vi. }(S \mid T) & \equiv(\text { vi.S }) \mid T, \quad i \notin f n(T) \\
\frac{S \longrightarrow T}{R|S \longrightarrow R| T} & \frac{S \longrightarrow T}{v i . S \longrightarrow v i . T} & \frac{S \equiv S^{\prime} \longrightarrow T^{\prime} \equiv T}{S \longrightarrow T}
\end{array}
$$

Fig. 4: Structural congruence and structural transitions.

$$
\begin{aligned}
& \langle\mathscr{E}[M]\rangle_{p} \longrightarrow\langle\mathscr{E}[V]\rangle_{p}, \text { if } M \Downarrow V \text { and } M \not \equiv V \\
& \langle\mathscr{E}[\operatorname{return} N>\rangle=M]\rangle_{p} \longrightarrow\langle\mathscr{E}[M N]\rangle_{p} \\
& \langle\mathscr{E}[\text { eval } M]\rangle_{p} \longrightarrow\langle\mathscr{E}[\text { return } V]\rangle_{p}, \text { if } M \Downarrow V \\
& \langle\mathscr{E}[\text { allNodes }]\rangle_{p} \longrightarrow\langle\mathscr{E}[\text { return } M]\rangle_{p} \text {, where } M \text { is an list of all nodes, starting with } p \\
& \left.\left.\langle\mathscr{E}[\operatorname{spawn} M]\rangle_{p} \longrightarrow v i .\left(\langle\mathscr{E}[\operatorname{return} i]\rangle_{p}|i\{\langle\langle M\rangle\rangle=\operatorname{rput} i\rangle\rangle_{\{p\}}\right\}_{p}|\langle\langle M\rangle\rangle=\operatorname{rput} i\rangle\right\rangle_{p}\right) \text {, where } i \notin f n(\mathscr{E}) \\
& \left.\left.\langle\mathscr{E}[\operatorname{spawnAt} q M]\rangle_{p} \longrightarrow v i .\left(\langle\mathscr{E}[\operatorname{return} i]\rangle_{p}|i\{\langle M\rangle\rangle=\operatorname{rput} i\rangle_{q}\right\}_{p}|\langle M\rangle\rangle=\operatorname{rput} i\right\rangle_{q}\right) \text {, where } i \notin f n(\mathscr{E}) \\
& \text { (spawnAt) } \\
& \langle\mathscr{E}[\operatorname{rput} i M]\rangle_{p} \mid i\left\{\langle N\rangle_{p}\right\}_{q} \longrightarrow\langle\mathscr{E}[\text { return }()]\rangle_{p} \mid i\{M\}_{q} \\
& \langle\mathscr{E}[\text { rput } i M]\rangle_{p} \mid i\left\{\langle\langle N\rangle\rangle_{Q}\right\}_{q} \longrightarrow\langle\mathscr{E}[\text { return }()]\rangle_{p} \mid i\{M\}_{q} \\
& \langle\mathscr{E}[\operatorname{rput} i M]\rangle_{p} \mid i\{N\}_{q} \longrightarrow\langle\mathscr{E}[\text { return }()]\rangle_{p} \mid i\{N\}_{q} \text {, where } N \neq \perp \\
& \langle\mathscr{E}[\operatorname{rput} i M]\rangle_{p} \mid i\{\perp\}_{q} \longrightarrow\langle\mathscr{E}[\text { return }()]\rangle_{p} \mid i\{\perp\}_{q} \\
& \langle\mathscr{E}[\text { get } i]\rangle_{p} \mid i\{M\}_{p} \longrightarrow\langle\mathscr{E}[\text { return } M]\rangle_{p} \mid i\{M\}_{p}, \text { where } M \neq \perp \\
& \langle\langle M\rangle\rangle_{p_{1}}\left|i\left\{\langle\langle M\rangle\rangle_{P}\right\}_{q} \longrightarrow\langle\langle M\rangle\rangle_{p_{2}}\right| i\left\{\langle\langle M\rangle\rangle_{P}\right\}_{q} \text {, if } p_{1}, p_{2} \in P \\
& \langle\langle M\rangle\rangle_{p}\left|i\left\{\langle\langle M\rangle\rangle_{P_{1}}\right\}_{q} \longrightarrow\langle\langle M\rangle\rangle_{p}\right| i\left\{\langle\langle M\rangle\rangle_{P_{2}}\right\}_{q} \text {, if } p \in P_{1} \cap P_{2} \\
& \langle\langle M\rangle\rangle_{p} \longrightarrow\langle M\rangle_{p} \\
& i\left\{\langle M\rangle_{q}\right\}_{p}\left|\operatorname{dead}_{q} \longrightarrow i\left\{\langle M\rangle_{p}\right\}_{p}\right|\langle M\rangle_{p} \mid \operatorname{dead}_{q}, \text { if } p \neq q \\
& i\left\{\langle\langle M\rangle\rangle_{Q}\right\}_{p}\left|\operatorname{dead}_{q} \longrightarrow i\left\{\langle\langle M\rangle\rangle_{\{p\}}\right\}_{p}\right|\langle\langle M\rangle\rangle_{p} \mid \operatorname{dead}_{q} \text {, if } p \neq q \text { and } q \in Q \\
& \operatorname{dead}_{p} \mid\langle\langle M\rangle\rangle_{p} \longrightarrow \operatorname{dead}_{p} \\
& \langle\text { return }()\rangle_{p} \longrightarrow \quad \text { (gc_thread) } \\
& v i . i\{\}_{p} \longrightarrow \quad \text { (gc_ivar) } \\
& \operatorname{dead}_{p} \mid\langle M\rangle_{p} \longrightarrow \operatorname{dead}_{p} \\
& \operatorname{dead}_{p}\left|i\{?\}_{p} \longrightarrow \operatorname{dead}_{p}\right| i\{\perp\}_{p} \\
& \longrightarrow \operatorname{dead}_{p}
\end{aligned}
$$

Fig. 5: Small-step semantics of HdpH-RS.

Figure 5 presents the transition rules for HdpH-RS. Most of these rules execute a thread, relying on an evaluation context $\mathscr{E}$ to select the first action of the thread's monadic computation. Rules that are similar to those in (Marlow et al., 2011) are not explained in detail.

The first three rules are standard for monadic DSLs; note how eval is just a strict return. The rule (allNodes) exposes a list of all the system's nodes, in no paricular order except that the current node is the head of the list. The rules (spawn) and (spawnAt) define the work distribution primitives. The primitive spawn creates an IVar $i$ on the current node $p$ and wraps its argument $M$, followed by a write to $i$, into a spark initially residing on $p$; it also stores the spark in the empty IVar, as backup for fault recovery. In contrast, spawnAt wraps $M$ into a thread, which is placed on node $q$ (and backed up in the empty IVar for fault recovery). The side condition on both rules ensures that the name $i$ is fresh, i.e. does not occur free in the current thread. The (rput_*) and (get) rules for IVars are similar to those 
in (Marlow et al., 2011) except that IVars in HdpH-RS can only be read on the node they reside on. They can however be written from any node, and writes can be raced 3 the first write wins and overwrites the backup thread/spark, subsequent writes have no effect. Rules (migrate), (track) and (convert) govern the fault tolerant scheduling of sparks. A spark may freely migrate from node $p_{1}$ to $p_{2}$, subject to both locations being tracked by set $P$. The tracking set may change via rule (track) in arbitrary ways, provided the current location of the spark remains a member, modelling the supervising IVar's changing and uncertain knowledge about the location of a supervised spark; Figure 10 in Section 5.4 shows how rules (migrate) and (track) abstract the actual behaviour of the HdpH-RS work stealing algorithm. Migrating sparks cannot be executed directly; instead rule (convert) must turn them into threads that can execute but not migrate. Finally, the $\left(\mathrm{gc}_{-}{ }^{*}\right)$ rules eliminate garbage, i.e. terminated threads and inaccessible IVars. Note that to become garbage, IVars must be unreachable, and sparks must be converted and executed to termination; hence the semantics does not support speculative parallelism.

The remaining rules deal with faults. The four rules at the bottom right of Figure 5 define the fault model of HdpH-RS. A node $p$ may die any time, signalled by the spontaneous production of $\operatorname{dead}_{p}$, and non-deterministically its sparks and threads may disappear and its IVars may turn into zombies. IVars cannot just disappear, or else writes to IVars on dead nodes would get stuck instead of behaving like no-ops. However, some of $p$ 's sparks and threads may survive and continue to execute. In this way the semantics models partial faults and pessimistic notification of faults. Node failure is permanent as no transition consumes $\operatorname{dead}_{p}$.

Finally, the (recover_thread) and (recover_spark) rules model the recovery of tasks that have been lost due to faults. A thread supervised by IVar $i$ on $p$ and executing on dead node $q \neq p$ is replicated on $p$, after which $i$ updates the tracking location to $p$ (which effectively rules out further supervision as there is no point supervising a thread on the same node). A spark supervised by IVar $i$ on $p$ and known to reside on some node in the tracking set $Q$ is replicated on $p$ if any node $q \in Q$ is dead; afterwards $i$ continues to supervise, now tracking $\{p\}$, the location of the replica spark. Due to the inherent uncertainty of tracking, sparks may be replicated even when actually residing on healthy nodes.

The transition rules are illustrated by executions with and without faults in (Stewart, 2013b). Section 5.4 relates the operational semantics to the actual fault tolerant work stealing algorithm of HdpH-RS.

\subsection{Transparent Fault Recovery}

We aim to show that, under reasonable assumptions, fault recovery is transparent, i.e. semantically unobservable in the sense that it cannot change the result of reductions. We need to formally define a number of concepts, leading to the result of a reduction, namely a normal form of its initial state.

We note that in any reduction, rule (dead) permutes with every rule to the left. That is, in any given reduction it is irrelevant how early a failing node dies; what matters is the

3 Since the DSL in Section 3.1 does not expose rput, races only occur as a result of task replication by the fault tolerant $\mathrm{HdpH}-\mathrm{RS}$ scheduler. 
subsequent reaction, killing and/or recovering threads and sparks. Thus, for the purpose of investigating normal forms, we ban rule (dead) and instead start reduction from states of the form $S\left|\operatorname{dead}_{p_{1}}\right| \ldots \mid \operatorname{dead}_{p_{n}}$, insisting that all failing nodes die right at the start of the reduction. For the remainder of this section, let $F=\left\{p_{1}, \ldots, p_{n}\right\}$ be a set of failing nodes. We decorate the reduction relation $\longrightarrow$ with $F$ and define $S \longrightarrow_{F} T$ iff $S\left|\operatorname{dead}_{p_{1}}\right| \ldots \mid$ $\operatorname{dead}_{p_{n}} \longrightarrow T\left|\operatorname{dead}_{p_{1}}\right| \ldots \mid \operatorname{dead}_{p_{n}}$. We call $\longrightarrow \emptyset$ reductions failure-free; obviously, these reductions cannot use any of the rules (kill_*), (recover_*) and (rput_zombie).

A main thread is a thread of the form $\langle M\rangle_{p}$ with $f n(M)=\emptyset$, and a root thread is a thread of the form $\langle M>>=\text { rput } i\rangle_{p}$ with $f n(M)=\emptyset$. That is, a main thread has no free IVars, and a root thread has exactly one free IVar, which it only accesses to write its final result. A main thread corresponds to a program's initial thread (e.g. the thread executing the main action in a Haskell program) whereas root threads correspond to tasks created by spawn or spawnAt.

We call a state $S$ well-formed iff there is a state $S_{0}$ consisting of a single main or root thread such that $S_{0} \longrightarrow{ }_{F}^{*} S$, where $\longrightarrow{ }_{F}^{*}$ denotes the reflexive-transitive closure of $\longrightarrow F$. We observe that reductions starting from well-formed states cannot get stuck except when embedding the host language, namely term $M$ diverging in rules (normalize) and (eval), or when executing the final rput of the root thread. In particular, well-formedness guarantees that all other rputs find their target IVars, that all gets find their source IVars, and that these source IVars reside on the same nodes as the threads executing get.

Given a state $S$, a thread $\langle N\rangle_{p}$ is reachable from $S$ iff there is a state $T$ such that $S \longrightarrow{ }_{F}^{*} v i_{1} \ldots v i_{n} .\left(T \mid\langle N\rangle_{p}\right)$. Thread $\langle N\rangle_{p}$ is a normal form of $S$, denoted $S \downarrow_{F}\langle N\rangle_{p}$, iff $S \longrightarrow{ }_{F}^{*}\langle N\rangle_{p}$ and $\langle N\rangle_{p}$ is irreducible. If $F=\emptyset$ we call $\langle N\rangle_{p}$ a failure-free normal form. Note that reaching normal form entails $p \notin F$, otherwise $\langle N\rangle_{p}$ would be reducible by rule (kill_thread). Furthermore, reaching normal form entails that all parallel computation has ceased because threads and sparks have been garbage collected or killed (on failed nodes). Finally note that $S$ may have any number of normal forms.

We observe that the existence of normal forms allows compositional splicing of reductions. More precisely, given a state $S$ and a root thread $\left\langle M>>=\operatorname{rput} i_{k}\right\rangle_{p}$ such that $S \longrightarrow{ }_{F}^{*} v i_{1} \ldots v i_{n} \cdot\left(T \mid\left\langle M>>=\operatorname{rput} i_{k}\right\rangle_{p}\right)$ and $\left\langle M>>=\operatorname{rput} i_{k}\right\rangle_{p} \downarrow_{F}\langle N\rangle_{p}$, there is a reduction $S \longrightarrow{ }_{F}^{*} v i_{1} \ldots v i_{n} .\left(T \mid\langle N\rangle_{p}\right)$. Note that $f n\left(\langle N\rangle_{p}\right)=f n\left(\left\langle M>>=\operatorname{rput} i_{k}\right\rangle_{p}\right)=\left\{i_{k}\right\}$ due to the fact that $f n(M)=\emptyset$ and normalisation cannot create free IVars.

We have defined normal forms of arbitrary states $S$, yet we will mostly be interested in the normal forms of main and root threads, as the latter correspond to tasks spawned and potentially replicated. Ignoring potential divergence in rules (normalize) and (eval), reductions starting from main and root threads do not get stuck — see the above observation on well-formedness - hence existence (though not uniqueness) of normal forms is guaranteed. In particular, a normal form of a main thread $\langle M\rangle_{p}$ must be of the form $\langle\operatorname{return} N\rangle_{p}$ with $f n(N)=\emptyset$. Likewise, a normal form of a root thread $\langle M>>=\operatorname{rput} i\rangle_{p}$ must be of the form $\langle\text { rput } i N\rangle_{p}$ with $f n(N)=\emptyset$. Note that the name of the result IVar has no bearing on the normal forms of a root thread as $\langle M \gg>=\operatorname{rput} i\rangle_{p} \downarrow_{F}\langle\operatorname{rput} i N\rangle_{p}$ if and only if $\langle M>>=\operatorname{rput} j\rangle_{p} \downarrow_{F}\langle\operatorname{rput} j N\rangle_{p}$ for all IVars $i$ and $j$. However, HdpH-RS is a location-aware DSL, so moving a thread from node $p$ to $q$ may substantially alter its normal forms. 
We aim to transform reductions with failures into failure-free reductions, preserving normal forms. This isn't possible in general; it does require some restriction on the use of location information. We call a thread $\langle M\rangle_{p}$ location invariant iff it does not matter where it executes; that is, $\langle M\rangle_{p} \downarrow_{F}\langle N\rangle_{p}$ if and only if $\langle M\rangle_{q} \downarrow_{F}\langle N\rangle_{q}$ for all sets of nodes $F$ and all nodes $p$ and $q$. We call $\langle M\rangle_{p}$ transitively location invariant iff $\langle M\rangle_{p}$ is location invariant and all root threads $\langle N>>=\text { rput } j\rangle_{q}$ reachable from $\langle M\rangle_{p}$ are location invariant.

Now we can state the claim of the main theorem below. The normal forms of a transitively location invariant main thread coincide with its failure-free normal forms. That is, the effects of failure and recovery cannot be observed in the results of reductions (provided the main thread executes on a non-failing node).

Theorem 1 Let $F$ be a set of failing nodes and $\langle M\rangle_{p}$ a transitively location invariant main thread with $p \notin F$. Then for all normal forms $\langle N\rangle_{p}$, we have $\langle M\rangle_{p} \downarrow_{F}\langle N\rangle_{p}$ if and only if $\langle M\rangle_{p} \downarrow_{\emptyset}\langle N\rangle_{p}$.

Proof sketch. The reverse implication is trivial as the failure-free reductions are a subset of all possible reductions.

Proving the forward implication is done by splicing together reductions normalising the root threads arising from calls to spawn and spawnAt in the body of $M$. The root thread reductions in question are failure-free according to Lemma 2 below. Hence an argument similar to the step case in the proof of Lemma 2 yields the conclusion that there is a (spliced) failure-free reduction from $\langle M\rangle_{p}$ to $\langle N\rangle_{p}$.

Lemma 2 Let $F$ be a set of failing nodes, and let $\langle M>>=\operatorname{rput} i\rangle_{p}$ be a transitively location invariant root thread with $p \notin F$. Then $\langle M>>=\operatorname{rput} i\rangle_{p} \downarrow_{F}\langle\operatorname{rput} i N\rangle_{p}$ implies $\langle M>>=\operatorname{rput} i\rangle_{p} \downarrow_{\emptyset}\langle\operatorname{rput} i N\rangle_{p}$.

Proof sketch. We prove the claim by induction on the number of calls to spawn and spawnAt occuring during the normalisation of $\langle M>>=\text { rput } i\rangle_{p}$.

- Base case. Suppose the reduction from $\langle M>>=\operatorname{rput} i\rangle_{p}$ to $\langle\operatorname{rput} i N\rangle_{p}$ involves no calls to spawn and spawnAt. Then all states along the reduction are single threads of the form $\left\langle M^{\prime}\right\rangle>=$ rput $\left.i\right\rangle_{p}$, so none of the rules (recover_*) and (kill_*) apply. Hence the reduction is already failure-free.

- Step case. Suppose the reduction from $\langle M>>=\operatorname{rput} i\rangle_{p}$ to $\langle\operatorname{rput} i N\rangle_{p}$ is already failure-free up to a state $S$ of the form $\left.S=v i_{1} \ldots v i_{n} .\left(T\left|\left\langle\operatorname{spawn} M^{\prime}\right\rangle\right\rangle=M^{\prime \prime}\right\rangle_{p}\right)$. Rule (spawn) will create a new IVar $i_{n+1}$ and spark $\left.\left.\left\langle\left\langle M^{\prime}\right\rangle\right\rangle=\operatorname{rput} i_{n+1}\right\rangle\right\rangle_{p}$ on nonfailing node $p$. The spark may migrate, get converted to a root thread, get killed, be recovered, migrate, get killed, and so on. Eventually though, the IVar $i_{n+1}$ will be filled by some thread $\left\langle\operatorname{rput} i_{n+1} N^{\prime}\right\rangle_{q}$, which implies that $\left\langle\operatorname{rput} i_{n+1} N^{\prime}\right\rangle_{q}$ is a normal form of the root thread $\left.\left\langle M^{\prime}\right\rangle>=\operatorname{rput} i_{n+1}\right\rangle_{q}$. We may assume that $q$ is not a failing node, for otherwise we could use location invariance to transplant the normalisation of $\left\langle M^{\prime}>>=\operatorname{rput} i_{n+1}\right\rangle_{q}$ to a another non-failing node. Thus, we have $q \notin F$ and $\left.\left\langle M^{\prime}\right\rangle>=\operatorname{rput} i_{n+1}\right\rangle_{q} \downarrow_{F}\left\langle\text { rput } i_{n+1} N^{\prime}\right\rangle_{q}$, from which we get $\left.\left\langle M^{\prime}\right\rangle>=\operatorname{rput} i_{n+1}\right\rangle_{q} \downarrow_{\emptyset}$ $\left\langle\text { rput } i_{n+1} N^{\prime}\right\rangle_{q}$ by induction hypothesis. Therefore we can extend the above failurefree reduction $\langle M>>=\text { rput } i\rangle_{p} \longrightarrow_{\emptyset}^{*} S$ from state $S$ to a state $S^{\prime}$ of the form $S^{\prime}=$ 
$v i_{1} \ldots v i_{n+1} .\left(T\left|i_{n+1}\left\{N^{\prime}\right\}_{p}\right|\left\langle M^{\prime \prime}\right\rangle_{p}\right)$ by applying rule (spawn), followed by (track) and (migrate) to move the spark from $p$ to $q$, followed by (convert) and the splicedin failure-free reduction of $\left.\left.\left\langle M^{\prime}\right\rangle\right\rangle=\operatorname{rput} i_{n+1}\right\rangle_{q}$ to normal form, followed by rule (rput_empty_spark).

The argument for extending failure-free reductions over calls to spawnAt is similar if a little simpler because tasks created by spawnAt do not migrate and are recovered at most once. The claim follows by repeating the construction, eventually yielding a failure-free reduction from $\langle M>>=\operatorname{rput} i\rangle_{p}$ to $\langle\operatorname{rput} i N\rangle_{p}$.

Observations. Firstly, our definition of (transitive) location invariance is in terms of reductions, i.e. rests on the operational semantics. However, there are many cases where location invariance is guaranteed statically. For instance, skeletons like pushMapSliced (Listing 3 use location information only for scheduling decisions; in particular, locations are never written to IVars and never compared. Thus, the resulting skeletons are clearly location invariant. We leave a more thorough static characterisation of location invariance, e.g. in the form of a type system, for future work.

Secondly, for the purpose of presenting a simple semantics, we have ignored all observable effects apart from locations, and location invariance took care of reconciling the effects with task replication. A DSL with more realistic effects (e.g. tasks performing IO) would have to take more care. On top of location invariance, effects would need to be idempotent, i.e. invariant under replication, in order to guarantee the unobservability of failure and recovery.

Finally, HdpH-RS is a non-deterministic DSL as decisions taken by the non-deterministic scheduler may become observable, e.g. in case migrating tasks query their current location. However, the sublanguage that restricts HdpH-RS task distribution to spawnAt only is deterministic, due to entirely deterministic scheduling. Whether there are more interesting deterministic sub-languages, in the face of truly non-deterministic scheduling, is an interesting and timely (Kuper et al., 2014) open question.

\section{Reliable Work Stealing Protocol}

\subsection{Work Stealing Protocol}

The HdpH-RS fault tolerant work stealing protocol adds resilience to sparks and threads and involves a victim, a thief and a supervisor. A supervisor is the node where a supervised spark was created, and is responsible for guaranteeing the execution of that spark. A thief is a node with few sparks to execute. A victim is a node that may hold sparks and is targeted by a thief. The HdpH-RS runtime system messages serve two purposes: to schedule sparks from heavily loaded nodes to idle nodes, and to allow supervisors to track the location of sparks as they migrate between nodes.

The message handlers that implement the fishing protocol are in Section 4.4. A successful work stealing attempt is illustrated in Figure 6 . A thief node $\mathrm{C}$ targets a victim node $\mathrm{B}$ by sending a FISH message. The victim requests a scheduling authorisation from the supervisor with REQ. The supervisor grants authorisation with AUTH, and a spark is 


\begin{tabular}{|c|c|c|c|}
\hline Message & From & To & Description \\
\hline FISH thief & $\mathrm{T}$ & $\mathrm{V}$ & Fishing request from a thief. \\
\hline SCHEDULE spark victim & $\mathrm{V}$ & $\mathrm{T}$ & Victim schedules a spark to a thief. \\
\hline NOWORK & $\mathrm{V}$ & $\mathrm{T}$ & $\begin{array}{l}\text { Response to FISH: victim informs thief that it either does not hold } \\
\text { a spark, or was not authorised to schedule a spark. }\end{array}$ \\
\hline $\mathrm{REQ}$ ref seq victim thief & $\mathrm{V}$ & $\mathrm{S}$ & Victim requests authorisation to send spark to thief. \\
\hline DENIED thief & $\mathrm{S}$ & $\mathrm{V}$ & Supervisor denies a scheduling request with respect to REQ. \\
\hline AUTH thief & S & V & Supervisor authorises a scheduling request with respect to REQ. \\
\hline OBSOLETE thief & $\mathrm{S}$ & $\mathrm{V}$ & $\begin{array}{l}\text { In response to REQ: the task waiting to be scheduled by victim is an } \\
\text { obsolete task copy. Victim reacts to OBSOLETE by discarding task } \\
\text { and sending NOWORK to thief. }\end{array}$ \\
\hline ACK ref seq thief & & & Thief sends an ACK to the supervisor of a spark it has received. \\
\hline DEADNODE node & \multicolumn{2}{|c|}{ any node to itself } & A message from a node to itself reporting failure of a remote node. \\
\hline
\end{tabular}

Table 1: HdpH-RS Messages

scheduled from the victim to the thief in a SCHEDULE message. When the thief receives the SCHEDULE it sends an ACK to the supervisor.

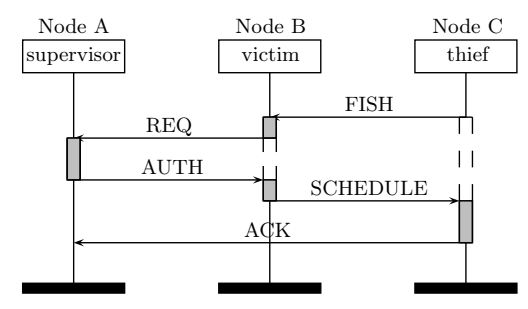

Fig. 6: Fault Tolerant Fishing Protocol in HdpH-RS

The HdpH-RS Runtime System (RTS) messages are described in Table1. The Message column is the message type, the From and To columns distinguish a supervisor node (S), a thief node $(\mathrm{T})$ and a victim node $(\mathrm{V})$. The Description column shows the purpose of the message. The use of each message are described in the scheduling algorithms in Section 4.4

\subsection{Task Locality}

For the supervisor to determine whether a spark is lost when a remote node has failed, the migration of a spark needs to be tracked. This is made possible by the RTS messages REQ and ACK. An example of task migration tracking is in shown in Figure 7 . Node A is supervising spark 0 . The messages REQ and ACK are received by the supervising node A to keep track of a spark's location. Sparks and threads can therefore be identified by their corresponding IVar.

The IVar data structure in Hdph-RS plays an important role for fault tolerance, shown in Listing 4 . Not only are they used to implement futures, they also store supervision information about the corresponding task. An empty IVar keeps track of the get calls from other threads in blockedThreads, and supervision state in supervisedState. The 


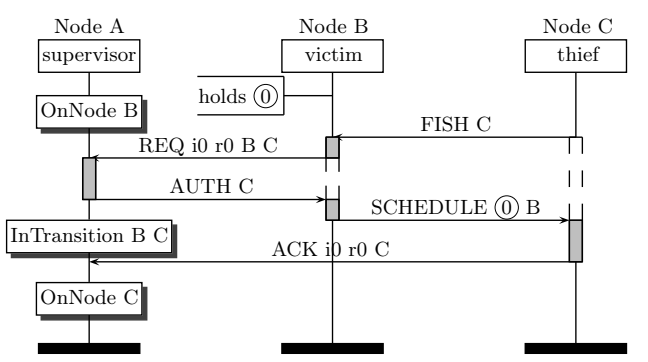

Fig. 7: Migration Tracking with Message Passing

SupervisedTaskState keeps a local copy of the corresponding task in task, a task replica count in replicaNum and the known task location in location - either OnNode node or InTransition [node].

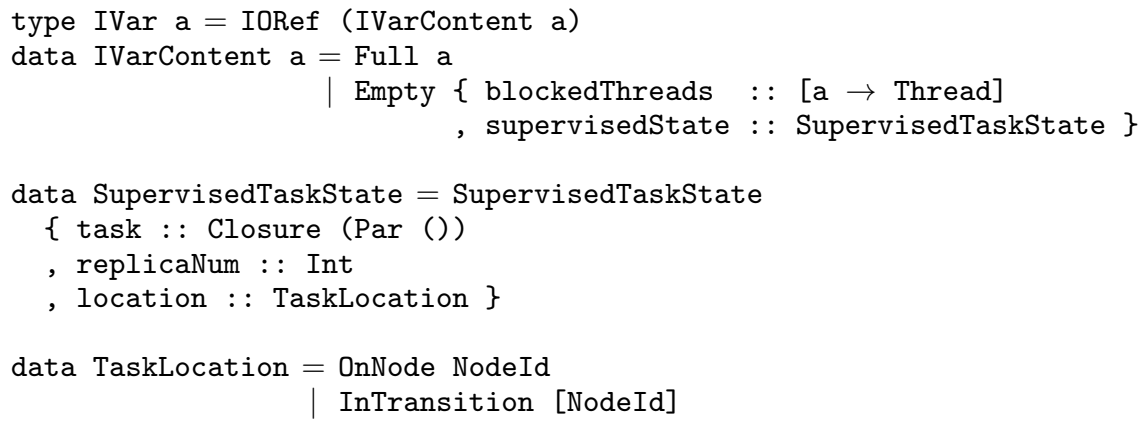

Listing 4: Holding Supervision State in Empty IVars

The message REQ is used to request authorisation to schedule the spark to another node. If the supervisor knows that it is in the sparkpool of a node (i.e. OnNode thief) then it will authorise the fishing request with AUTH. If the supervisor believes it is in-flight between two nodes then it will deny the request with DENIED. Examples of unsuccessful work stealing attempts are given in (Stewart, 2013b).

\subsection{Duplicate Sparks}

Location tracking for a task switches between two states in the corresponding empty IVar (Listing 4), either OnNode if the supervisor receives an ACK or InTransition if the supervisor authorises a migration in response to a REQ. To ensure the safety of sparks, the scheduler makes pessimistic assumptions that tasks have been lost when a node fails. If a supervisor is certain that a spark was on the failed node, then it is replicated. If a supervisor believes a spark to be in-flight either towards or away from the failed node during a fishing operation, again the spark is replicated. The consequence is that the scheduler may create duplicates.

Duplicates of the same spark can co-exist in a distributed environment with one constraint. Older obsolete spark replicas are not permitted to migrate through work stealing, 


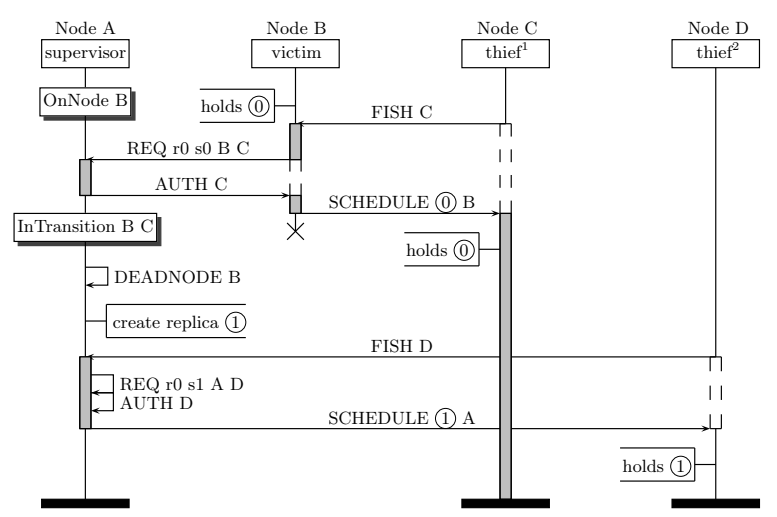

Fig. 8: Pessimistic Scheduling that Leads to Spark Duplication

as multiple migrating copies with the same reference may potentially lead to inconsistent location tracking. However, they are permitted to transmit results to IVars using rput. Thanks to idempotence, this scenario is indistinguishable from the one where the obsolete replica has been lost. This possibility is illustrated in Figure 8. A spark is created with spawn on node A and then fished by node B. B fails during a fishing exchange between $\mathrm{B}$ and $\mathrm{C}$, and $\mathrm{A}$ receives a DEADNODE notification of the failure of $\mathrm{B}$. At this point, supervising node A has not yet received an ACK from C, and pessimistically replicates the spark locally. In this instance the original spark survived the failure of node B and there are now two copies of the spark. This is a partial execution, so neither nodes C or D send an ACK in this message sequence to return the spark supervision state to OnNode on node A.

\subsection{Fault Tolerant Scheduling Algorithm}

The message handlers that implement the fault tolerant algorithm are in Algorithm 1 Each node is proactive in their search for work with fishing, triggering a sequence of messages between the victim, thieving and supervising nodes. The FISH handler (line 11) shows how a node that receives a FISH has been targeted by a thief. The victim checks that this node has not already been targeted by another thief (line 2 ) and is waiting for authorisation. If it is, then a NOWORK reply is sent to the thief. Otherwise, if the victim has an available spark then it is reserved and the victim sends a REQ to the supervisor. If the sparkpool is empty, a NOWORK is sent to the thief.

The REQ handler is shown on line 11. If the location of the reserved spark is known precisely by the supervisor, the request is granted with AUTH. Otherwise, if the task is believed to be in transition between two nodes, the request is rejected with DENIED. If the spark is obsolete, then the victim is instructed to discard it with an OBSOLETE message.

A victim's AUTH handler is shown on line 21. It sends the reserved spark to a thief in a SCHEDULE message on line 22. The handler for scheduled sparks is shown on line 23. A thief adds the spark to its own sparkpool (line 24), and sends an acknowledgement of its arrival to its supervisor (line 25). However, if a victim is informed that the reserved spark is an obsolete copy with OBSOLETE from the supervisor, it discards the reserved spark and 


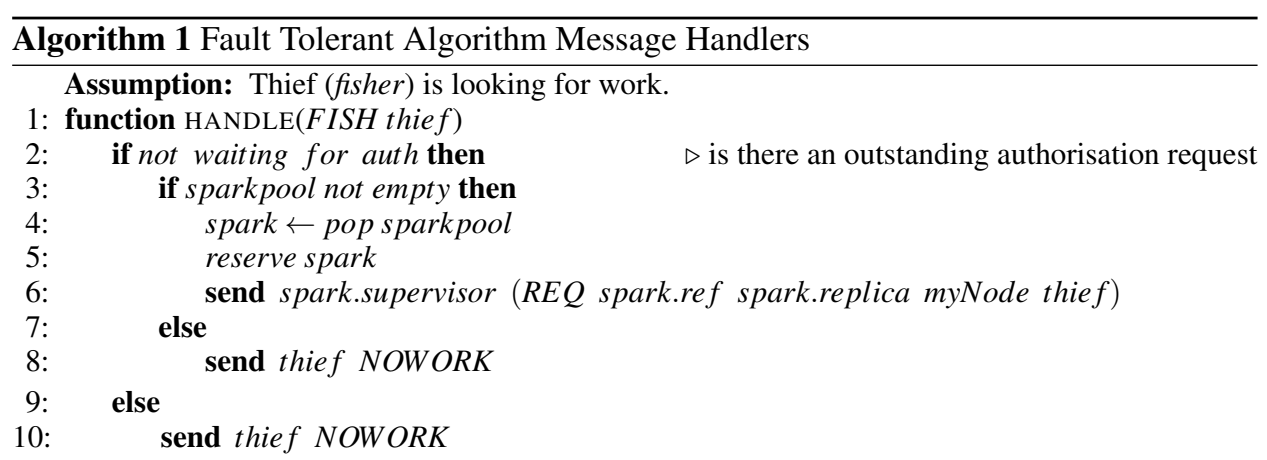

Assumption: A schedule request is sent from a victim to this supervising node.

11: function $\operatorname{HANDLE}(R E Q$ ref replica victim thief $)$

12: if replicaOfref $==$ replica then $\quad \triangleright$ remote task is most recent copy

location $\leftarrow$ location $O f$ ref

14: $\quad$ if location $==$ OnNode then

15: $\quad$ update location (InTransition victim thief)

16: $\quad$ send victim (AUTH thief)

17: $\quad$ else if location $==$ InTransition then

18: $\quad$ send victim (DENIED thief)

else

send victim (OBSOLETE thief) $\quad \triangleright$ task is old copy, order victim to discard

$\triangleright$ task location known

$\triangleright$ authorise the request

$\triangleright$ deny the request

Assumption: Location state on supervisor was OnNode.

21: function HANDLE(AUTH ref thief)

22: send thief (SCHEDULE reserved_spark) D send thief the spark

Assumption: A Victim was authorised to send this node a spark in a SCHEDULE.

23: function HANDLE(SCHEDULE spark)

24: insert spark sparkpool $\quad \triangleright$ add spark to sparkpool

25: $\quad$ send spark.supervisor (ACK spark.ref spark.replica myNode)

Assumption: Thief receives a spark.

26: function HANDLE( $A C K$ ref thief)

27: update (locationOf ref) (OnNode thief) ) set spark location to OnNode

Assumption: A remote node has died.

28: function HANDLE(DEADNODE deadNode)

29: $\quad$ if fishVictim $==$ deadNode then

30: $\quad$ resume fishing

31: $\quad$ if thiefOfGuardedSpark $==$ deadNode then

32: $\quad$ insert reserved_spark sparkpool

33: $\quad$ for all $s \in$ sparks on deadNode do

34: $\quad$ insert $s$ sparkpool

$\triangleright$ return reserved spark to sparkpool

insert $s$ sparkpool

35: $\quad$ insert $t$ threadpool $\quad \triangleright$ replicate potentially lost thread: convert \& execute locally

sends a NOWORK message to the thief. The pseudo code for the OBSOLETE and DENIED message handlers is in (Stewart, 2013b).

The supervisor handler for ACK messages is shown on line 26. It updates the migration tracking for this spark to OnNode, a state that will allow another thief to steal from the new host of the spark. 
The handler for DEADNODE messages is on line 28 There are four checks performed by every node when a remote node fails. First, it checks if it is waiting for a fishing reply from the dead node (line 29. Second, whether the dead node is the thief of the spark it has requested authorisation for (line 31). Third, it identifies the sparks are at-risk due to the remote node failure (line 33). Fourth, it identifies the threads are at-risk due to the remote node failure (line 35). All at-risk sparks are replicated and added to the local sparkpool. These duplicates can be fished again for load-balancing (line 34). All at-risk threads are replicated and are converted and executed locally (line 36.

\section{Validating Reliable Work Stealing}

\subsection{Desirable Scheduling Properties with Non-Deterministic Systems}

The SPIN model checker is used to ensure that the HdpH-RS scheduling algorithm honours the small-step semantics (Section 3.4), supervising sparks in the absence and presence of faults. Due to the many sources of non-determinism in faulty systems, it is easy to make mistakes in correctness arguments for fault tolerant distributed systems. Hence distributed faulty systems are natural candidates for model checking (John et al., 2013), and specifically because of two properties of HdpH-RS:

1. Asynchronous message passing Causal ordering (Lamport, 1978) of asynchronous distributed scheduling events is not consistent with wall-clock times. HdpH-RS message passing between nodes is asynchronous and non-blocking, instead writing to channel buffers. Because of communication delays, knowledge of remote node availability could be outdated (Xu \& Lau, 1997).

2. Work stealing To recover tasks in the presence of failure, a supervisor must be able to detect node failure and must always know the location of its tasks. The asynchronous message passing for stealing work complicates location tracking. The protocol for reliably relocating tasks between nodes in the presence of failure is intricate (Section 4), and model checking the protocol increases confidence in the design.

The HdpH-RS reliable scheduling algorithm is abstracted into a Promela model. The supervisor and worker abstractions are in Appendix A, and the full model is available online (Stewart, 2013a). Promela (Holzmann, 2004) is a meta-language for building verification models, and the language features are intended to facilitate the construction of high-level abstractions of distributed systems. Temporal logic (Prior, 1957) provides a formalism for describing the occurrence of event in time that is suitable for reasoning about concurrent programs (Pnueli, 1977). The SPIN model checker is used to verify key reliable scheduling properties of the algorithm design from Section 4.4, expressed in linear temporal logic (LTL). The properties are:

1. The IVar is empty until a result is sent: Evaluating a task involves transmitting a value to the supervisor, the host of the IVar associated with the task. This property verifies that the IVar cannot be full until one of the nodes has transmitted a value to the supervisor. 
2. The IVar is eventually always full: The IVar will eventually be filled by either a remaining worker, or the supervisor. This is despite the failure of any or all worker nodes.

The LTL properties guarantee spark evaluation, i.e. the associated future (IVar) on the supervising node is eventually filled. A counter property is used to ensure the Promela abstraction does model potential failure of any or all of the worker nodes. It checks that the model potentially kills one or more mortal workers, and SPIN is used to find counterexample executions when one of the workers terminates.

\subsection{HdpH-RS Abstraction}

Promela programs consist of processes, message channels, and variables. Processes are global objects that represent the concurrent nodes in HdpH-RS. The HdpH-RS scheduler has been simplified to its core supervision behaviours that ensure task survival. The model considers tasks scheduled with spawn in HdpH-RS - as the location of threads scheduled with spawnAt is always known and would not elicit race conditions on location tracking REQ and ACK messages.

Scope The Promela model is an abstraction that encapsulates behaviours necessary for guaranteeing the evaluation of sparks. There are six characteristics of the HdpH-RS scheduler in the Promela model:

1. One supervisor that initially puts a spark into its local sparkpool. It also creates spark replicas when necessary (item 6). The supervisor does not die.

2. Three workers that attempt to steal work from the supervisor and each other. Failure of these nodes is modeled by terminating the Promela process for each node. The workers can die.

3. Computation of a spark may happen at any time by any node that holds a copy of the spark. This simulates the execution of the spark, which would eventually invoke an rput call to fill the IVar on the supervisor. It is modeled by sending a RESULT message to the supervisor.

4. Failure of a worker node means that future messages to it are lost. The (dead) transition rule is modelled with a non-deterministic suicidal choice any of the three worker nodes can make. This choice results in a node asynchronously broadcasting its death to the remaining healthy nodes and then terminating. Failure detection is modeled by healthy nodes receiving DEADNODE messages.

5. Asynchronicity of both message passing and failure detection is modeled in Promela using buffered channels. Buffered channels model the buffered FIFO TCP connections in HdpH-RS.

6. Replication is used by the supervisor to ensure the safety of a potentially lost spark in the presence of node failure. The model includes spark replication from Section 4.4, honouring the (recover_spark) small-step transition rule in Section 3.4 Replication numbers are used to tag spark replicas in order to identify obsolete spark copies. Obsolete replica migration could potentially invalidate location records for a spark. Therefore, victims are asked to discard obsolete sparks. 
Termination of the scheduling algorithm is enforced in the model by aging the spark through transitions of the model. The age of the spark is zero at the initial system state. Each time it is scheduled to a node, its age is incremented. Moreover, each time it must be replicated by the supervisor its age is again incremented. When the age of the spark reaches 100, it is executed immediately. This models the HdpH-RS assumption that a scheduler will eventually execute the spark in a sparkpool and send a result to an empty IVar with an rput call.

Some aspects of the HdpH-RS scheduler design and implementation are abstracted away in the Promela model, because they are not part of the fault tolerance mechanism. The model involves only one IVar and one spark, which may manifest into multiple replicas - one active and the rest obsolete. Multiple IVars are not modeled, nor are threads created with spawnAt.

\subsection{Scheduling Model}

Nodes interact with message passing: ! to send and ? to receive. The channels in the Promela model of HdpH-RS are asynchronous, so that messages can be sent to a channel buffer, rather than being blocked waiting on a synchronised participatory receiver. This reflects the data buffers in the underlying TCP sockets and the asynchronous HdpH-RS model.

Supervisor Node The supervisor is modeled as an active proctype, so is instantiated in the initial system state. The supervisor executes a repetitive control flow that receives work stealing messages from worker nodes and authorisation messages from the supervisor, shown in Listing A 1. It creates the initial spark copy and then initiates the three workers. The underlying automaton is a message handling loop from SUPERVISOR_RECEIVE. The exception is when the spark has migrated 100 times the supervisor sends a RESULT message to itself to force termination. The label SUPERVISOR_RECEIVE is re-visited after the nondeterministic message handling choice, and is only escaped if a RESULT message has been received. In this case the IVar becomes full and the supervisor terminates.

Worker Nodes Each worker executes a repetitive control flow that receives work stealing message from worker nodes and authorisation messages from the supervisor, shown in Listing A 2. The underlying automaton is a message handling loop from WORKER_RECEIVE.

The exception is when the spark has migrated 100 times a RESULT message is sent to the supervisor. Otherwise the control flow takes one of three non-deterministic choices: the node may die; it may send a RESULT message to the supervisor if it holds a replica; it may receive a work stealing message from a work or scheduling request response from the supervisor. The WORKER_RECEIVE label is re-visited after the non-deterministic message handling choice, and is only escaped if it has died or the IVar on the supervisor is full. In either case the worker terminates.

The HdpH-RS transport layer sends DEADNODE messages to the scheduler message handler on each node when a connection with a remote node is lost. Failure is modeled by a non-deterministic choice that worker nodes can make whilst waiting for messages to arrive. A node can choose to die, which triggers the sending of a DEADNODE message to all 
thief keeps track of which nodes it has failed to steal a spark from and does not repeat the fishing request to these nodes (Stewart, 2013b).

\subsubsection{Spark Location Tracking}

When the task tracker records on the supervisor is ONNODE, then it can be sure that the node identified with spark.location.at is holding the spark. If the node fails at this point, then the spark should be recreated as it has certainly been lost. However, when a spark is in transition between two nodes i.e INTRANSITION, the supervisor cannot be sure of the location of the spark; it is on either of the nodes identified by spark.location.from or spark.location.to. To overcome this uncertainty, the model faithfully reflects the HdpH-RS pessimistic duplication strategy when a DEADNODE is received. This potentially generates replicas that concurrently exist in the model and they are handled using replica counts (Section 4.3).

\subsection{Correspondence with Operational Semantics}

An example of location tracking with the migrate_spark rule (Section 3.4) is shown in Figure 10 It is a more detailed version of the fault tolerant fishing protocol from Figure 7 The Promela message passing syntax in the message sequence diagram is an abstraction of send and receive Haskell function calls in the HdpH-RS implementation. Node B is the victim, and node $\mathrm{C}$ is the thief. The states for the IVar and the spark are $i\left\{\langle\langle M\rangle\rangle_{\{B\}}\right\}_{A}$ and $\langle\langle M\rangle\rangle_{B}$, showing that the supervisor $A$ holds IVar $i$ and that its corresponding spark is on node $B$. The (track) rule is fired before sending AUTH to the victim. The (migrate) rule is fired when the thief receives the SCHEDULE message containing the spark. The (track) rule is fired when the supervisor receives an ACK message from the thief. Once this message sequence is executed, the new states for the IVar and the spark are $i\left\{\langle\langle M\rangle\rangle_{\{C\}}\right\}_{A}$ and $\langle\langle M\rangle\rangle_{C}$.

\subsection{Model Checking Results}

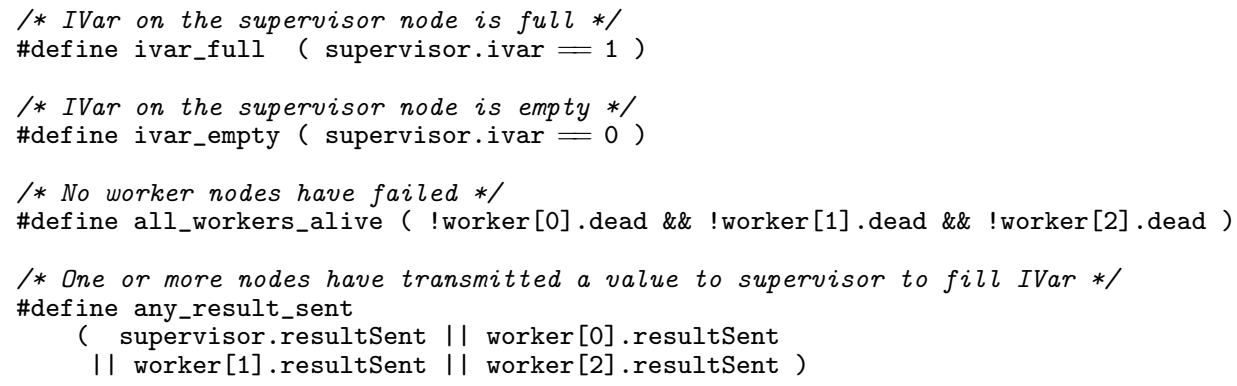

Listing 5: Propositional Symbols used in LTL Formulae of Fault Tolerant Properties

LTL is used to reason about causal and temporal relations of the HdpH-RS scheduler properties. The propositional symbols used in the verification of scheduling Promela model 


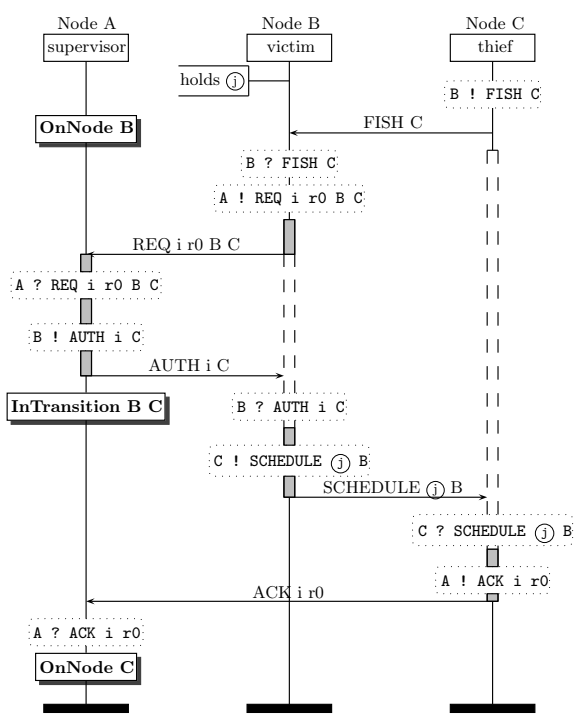

(a) Message sequence

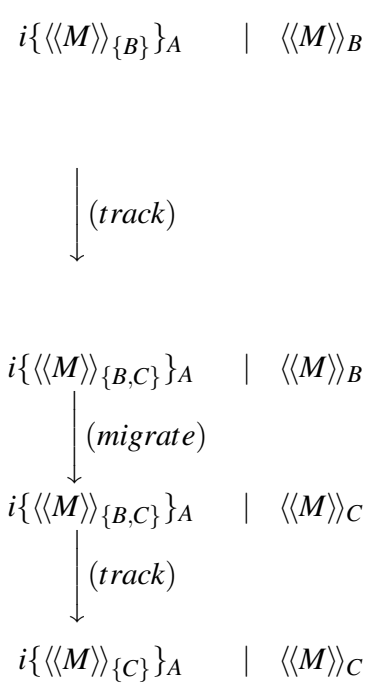

(b) State transitions

Fig. 10: Location Tracking with (migrate) and (track) rules

\begin{tabular}{lccccc}
\hline LTL Formula & Counter property & Depth & States & Transitions & Memory \\
\hline$\square$ all_workers_alive & Yes & 11 & 5 & 5 & $0.2 \mathrm{Mb}$ \\
$\square($ ivar_emptyU any_result_sent $)$ & No & 124 & $3.7 \mathrm{~m}$ & $7.4 \mathrm{~m}$ & $83.8 \mathrm{Mb}$ \\
$\diamond \square$ ivar_full & No & 124 & $8.2 \mathrm{~m}$ & $22.4 \mathrm{~m}$ & $84.7 \mathrm{Mb}$ \\
\hline
\end{tabular}

Table 2: Model Checking Results

are shown in Listing 5. The results of model checking the three LTL properties are in Table 2 Taking the $\diamond \square$ ivar_full property as an example, the results can be interpreted as follows. A reachable depth of 124 is found by SPIN for the model. The reachable state space is 8.2 million. A total of 22.4 million transitions were explored in the search. Actual memory usage for states was $84.7 \mathrm{Mb}$.

The IVar is Empty Until a Result is Sent To check that the model is faithful to the fact that an IVar is empty at least until its corresponding task has been evaluated, the $\square$ (ivar_empty $U$ any_result_sent) formula is verified. SPIN searches for two violating system states. First, where ivar_empty is false before any_result_sent is true. Second, a cycle of identical system states is identified while any_result_sent remains false. This is due to the nature of the strong until connective stipulating that the symbol any_result_sent must eventually be true in some future state. SPIN cannot find a violating system state after exhaustively searching 3.7 million reachable states up to a depth of 124 . 
The IVar is Eventually Always Full The key property for fault tolerance is that the IVar on the supervisor must eventually always be full. This would indicate that either a worker node has sent a RESULT message, or the supervisor has written to the IVar locally. To verify, SPIN searches for a system state cycle when ivar_full remains false i.e. supervisor . ivar $==0$.

This LTL property checks for the fatal scenario when the supervisor does not replicate the spark in the model, when instead it must ensure the existence of at least one replica. This would happen if the HdpH-RS fault tolerant fishing protocol algorithm (Section 4) did not correctly track spark location. This may cause a supervisor to ignore DEADNODE message when it instead should replicate a spark, which would mean no RESULT message would be sent to the supervisor - a cause of deadlock in the HdpH-RS implementation. To verify SPIN searches for states where the spark_count value is 0 for all nodes, and the supervisor does not create a replica in any future state. SPIN cannot find a violating system state after exhaustively searching 8.2 million reachable states up to a depth of 124 .

Validating the Possibility of Worker Node(s) Fail To check that worker nodes are able to fail in the model, a verification attempt is made on the $\square$ all_workers_alive LTL formula. To check that the model has the potential to kill mortal workers, SPIN searches for a counter-example system state with any of the worker[0].dead, worker[1].dead or worker[2].dead fields set to true. SPIN trivially identifies a counter example after searching 5 system states by executing the choice to kill a node.

\section{Implementation}

\subsection{HdpH-RS Architecture}

The HdpH-RS architecture is closely based on the HdpH architecture (Maier \& Trinder, 2012), and both share some components with Cloud Haskell as outlined in Section 2. The architecture supports semi-explicit parallelism with work stealing, message passing and the remote writing to IVars. The reliable scheduler is an implementation of the design from Section 4 . Inter-node communication is abstracted into a communication layer, that provides startup and shutdown functionality, node IDs, and peer-to-peer message delivery between nodes. The communication layer detects and reports faults to the scheduler. Each node runs several thread schedulers, typically one per core. Each scheduler owns a dedicated threadpool that may be accessed by other schedulers for stealing work. Each node runs a message handler, which shares access to the sparkpool with the schedulers. In extending $\mathrm{HdpH}$, one module is added for the fault tolerant strategies, and 14 modules are modified. The fault detection, fault recovery and task supervision functionality amounts to approximately 2500 lines of Haskell code in HdpH-RS, and an increase of approximately $50 \%$ over $\mathrm{HdpH}$.

\subsection{Recovering Supervised Sparks and Threads}

When a supervisor receives a DEADNODE message indicating a node failure (Section 4.4), it may replicate tasks if their survival is at risk. This is decided by the DEADNODE handler in Algorithm 1 of Section 4 It uses replicateSpark and replicateThread in Listing 6 


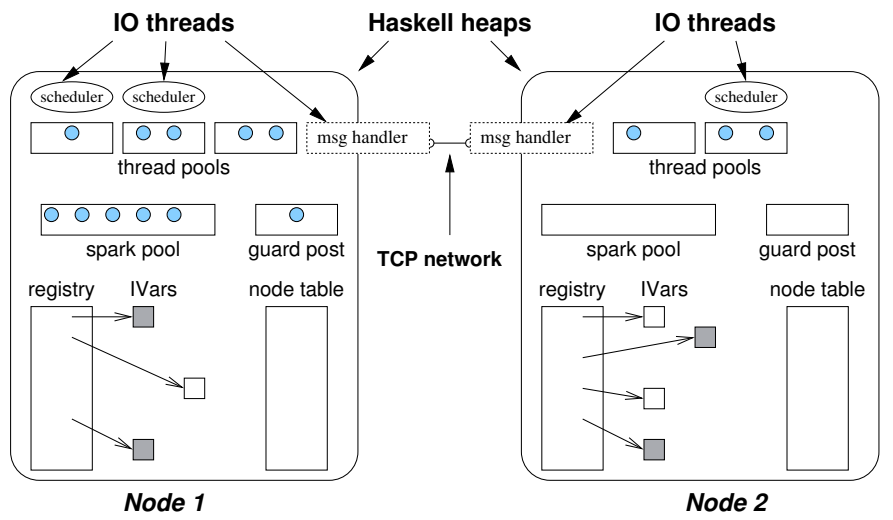

Fig. 11: HdpH-RS System Architecture

Both return a Maybe type, due to a potential race condition whereby another local scheduler or node writes a value to the IVar during the local recovery operation. If the IVar becomes full, then a Nothing value is returned indicating a full IVar and no recovery action needs taking.

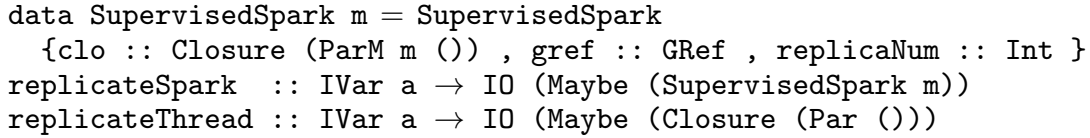

Listing 6: Replicating Sparks \& Threads in Presence of Failure

The replicateSpark and replicateThread functions both take an IVar as an argument. The DEADNODE handler has determined the safety of the corresponding task to be at risk as a consequence of node failure. Globalised IVar references are used to match remote tasks with locally hosted futures. The DEADNODE handler increments the replication number replicaNum in the IVar. A new task is created from a copy of the lost task (stored in SupervisedSpark as clo), and is scheduled according the recover_spark and recover _thread transition rules in the operational semantics (Section c). If a spark is being recovered, a SupervisedSpark is returned and added to the local sparkpool. If a thread is being recovered, a Closure ( $\operatorname{Par}()$ ) is returned, unpacked with unClosure, and added to a local threadpool.

\subsection{Fault Detecting Communications Layer}

Any fault in an MPI setting will typically bring down the entire MPI communicator, making this an unsuitable backend for fault. Instead, HdpH-RS uses a TCP-based transport layer, and nodes discover other nodes using UDP. The distributed virtual machine software stack is shown in Figure 12 .

TCP is an idle protocol, so if neither side sends any data once a connection has been established, then no packets are sent over the connection (Cleary, 2009). The act of receiving 


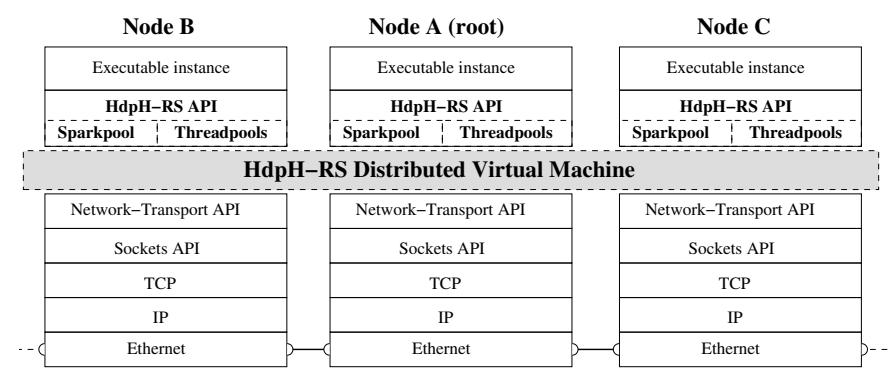

Fig. 12: HdpH-RS Distributed Virtual Machine Software Stack

data is completely passive in TCP, and an application that only reads from a socket cannot detect a dropped connection.

Two options are available for detecting failure with TCP. The first is to transmit keepalive messages over a connection. The second is to assume the worst, and implement timers for receiving messages on connections. If no packets are received within the timeout period, a connection may be regarded as lost. As architectures scale to thousands of nodes, error propagation through work stealing message transmissions cannot be relied upon. The HdpH-RS keep-alive messages is a guarantee of periodic traffic between nodes independent of work stealing messages, enabling nodes to discover failure by discovering lost connections.

Whilst the transmission of work stealing messages will probably trigger timely TCP failures for smaller architectures, there is a high failure detection latency in larger networks. This has an important implication for performance in HdpH-RS. Take an example where

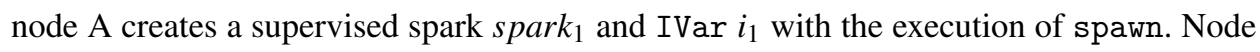
B fishes spark, and later suffers a power outage. Node A may not receive a TCP FIN message from B due to the sudden failure. Node A does not send any work stealing messages to $\mathrm{B}$, but is waiting for the value of evaluating spark to be written to $i_{1}$. To ensure a more reliable failure detection of $\mathrm{B}$, node $\mathrm{A}$ needs some other message transmission mechanism than just work stealing.

The keep-alive implementation in HdpH-RS is simple. As TCP failures are detected on send attempts, the keep-alive is silently ignored on the receiving end. After $N$ seconds, a node broadcasts a HEARTBEAT to all other nodes. When a node receives a HEARTBEAT message, it is silently ignored. For small architectures, heartbeats are unlikely to be the trigger that detects failure. On large architectures, work stealing messages between any two nodes are less likely to be transmitted within the keep-alive frequency, so the keep-alive messages are an important mechanism for failure detection. If a node detects the failure of another node, that remote node is removed from the list of peers returned by future calls to allNodes.

The main drawback to this failure detection strategy is the dependency on connection oriented protocols like TCP. There are two main weaknesses. First, the failure detection strategy of using connection-oriented transmission attempts would not work for connectionless protocols like UDP (Postel, 1980). Second, the design assumes a fully connected network. Every node has a persistent connection with every other node. The scalability limitations of TCP connections are well known (Ha et al., 2008). 


\subsection{HdpH and HdpH-RS Pragmatics}

In the design and semantics presented in Section 3 the fault tolerant spawn primitives have identical names to the $\mathrm{HdpH}$ primitives, making it trivial to switch between normal and fault tolerant execution. However in the current HdpH-RS implementation the fault tolerant primitives have different names, i.e. supervisedSpawn and supervisedSpawnAt, as do the HdpH-RS skeletons, e.g. parMapSlicedFT and pushMapSlicedFT. However, both primitives and skeletons preserve the same types, so switching between normal and fault tolerant versions of a program is a trivial alpha conversion. A more elegant solution would be to use the same name for the spawning primitives, and hence have identical skeleton implementations. Better still would be to integrate the $\mathrm{HdpH}$ and $\mathrm{HdpH}-\mathrm{RS}$ implementations, then programmers could switch between reliable and normal schedulers with a runtime flag.

\section{Evaluation}

\subsection{Measurement Platform}

Benchmarks The runtime performance of HdpH-RS is measured using the four benchmarks in Table 4 Existing Haskell parallel implementations are ported to HdpH-RS from GpH (Hammond et al., 2007) and monad-par (Marlow \& Newton, 2013) and parallelised using fault tolerant and non-fault tolerant skeletons. An open access dataset (Stewart et al., 2015) accompanies this paper, which includes the SPIN model (Section 5), scripts for collecting and plotting results and the Haskell source code for the following benchmarks.

\begin{tabular}{llllc}
\hline Benchmark & Skeleton & Code Origin & Regularity & code size (lines) \\
\hline Sum Euler & chunked parallel maps & HdpH & Some & 2 \\
Summatory Liouville & sliced parallel map & GUM & Little & 30 \\
N-Queens & divide-and-conquer & monad-par & Little & 11 \\
Mandelbrot & MapReduce & monad-par & Very little & 12 \\
\hline
\end{tabular}

Table 3: HdpH-RS Benchmarks

Sum Euler is a symbolic benchmark that sums Euler's totient function $\phi$ over long lists of integers. Sum Euler is an irregular data parallel problem where the irregularity stems from computing $\phi$ on smaller or larger numbers.

The Liouville function $\lambda(n)$ is the completely multiplicative function where $\lambda(p)=-1$ for each prime $p$. Summatory Liouville $L(n)$ denotes the sum of the values of the Liouville function $\lambda(n)$ up to $n$, where $L(n):=\sum_{k=1}^{n} \lambda(k)$.

The Mandelbrot set is the set of points on the complex plane that remains bounded to the set when an iterative function is applied to that. It consists of all points defined by the complex elements $c$ for which the recursive formula $z_{n+1}=z_{n}^{2}+c$ does not approach infinity when $z_{0}=0$ and $n$ approach infinity. A depth parameter is used to control the cost of sequential Mandelbrot computations. A higher depth gives more detail and subtlety in the final image representation of the Mandelbrot set (Boije \& Johansson, 2009). 
The N-Queens problem computes how many ways $n$ queens can be put on an $n \times n$ chessboard so that no 2 queens attack each other (Rivin et al., 1994). The implementation uses divide-and-conquer parallelism with an explicit threshold. An exhaustive search algorithm is used.

Hardware Platforms and Configurations The HdpH-RS benchmarks are measured on two platforms. The first is a Beowulf cluster and is used to measure supervision overheads, and recovery latency in the presence of simultaneous and random failure. Each Beowulf node comprises two Intel Xeon E5504 quad-core CPUs at 2GHz, sharing 12Gb of RAM. Nodes are connected via Gigabit Ethernet and run Linux CentOS $5.7 x 86$ 64. Benchmarks are run on up to $32 \mathrm{HdpH}-\mathrm{RS}$ nodes, one per Beowulf node, scaling up to 256 cores.

The second is HECToR, a national UK high-performance computing service. The HECToR compute hardware is contained in 30 cabinets and comprises 704 compute blades, each containing four compute nodes running Compute Node Linux. Each node has two 16 core AMD Opteron $2.3 \mathrm{GHz}$ Interlagos processors split into four NUMA regions, with $16 \mathrm{~Gb}$ memory. Benchmarks are run on up to $200 \mathrm{HdpH}-\mathrm{RS}$ nodes, one per NUMA region, scaling up to 1400 cores. Peer discovery with UDP is not supported on HECToR, so the HdpH-RS fault detecting TCP-based transport layer cannot be used. The MPI-based $\mathrm{HdpH}$ transport layer has been retrofitted in to HdpH-RS for the purposes of assessing the scalability of the supervised work stealing in HdpH-RS on HECToR in the absence of faults.

HdpH-RS nodes on both the Beowulf and HECToR have 8 cores, 7 of which are used by the HdpH-RS node. This is common practice to limit performance variation on sharedmemory nodes (Harris et al., 2005, Maier \& Trinder, 2012). For every data point, the mean of 5 runs are reported along with standard error.

\subsection{Performance With No Failure}

Figure 13 shows runtime and speedup graphs for Summatory Liouville, though Figures $13 \mathrm{a}$ and $13 \mathrm{c}$ omit runtimes on 1 core for readability. Table 4 summarises the relative speedups obtained in strong scaling for the benchmarks with and without fault tolerance. Strong scaling uses the same input on 1 core as on many cores, and relative speedup compares with the same, potentially-parallel, program running on a single core. A complete set of performance results is available in (Stewart, 2013b).

The speedup measurements compare eager and lazy, and normal and fault tolerant skeleton implementations of the benchmarks. On the Beowulf Sum Euler to 250k is computed with parMapSliced skeletons and a threshold of $1 \mathrm{k}$. Summatory Liouville is computed with parMapSliced skeletons to $200 \mathrm{~m}$ and a threshold of $500 \mathrm{k}$ on the Beowulf, and to $500 \mathrm{~m}$ with a threshold of $250 \mathrm{k}$ on HECToR. Mandelbrot is computed mapReduce skeletons with a dimension of $4096 \times 4096$ and a threshold of 4 and a depth of 4000 on the Beowulf, and threshold 4, depth 8000 on HECToR.

The results show that the overheads of fault tolerance are low in the absence of faults as the maximum speedups for the fault tolerant and normal versions are very similar. Eager scheduling consistently out performs lazy work distribution for these programs that have limited irregularity. The parallel efficiency with eager scheduling is approximately $50 \%$, 


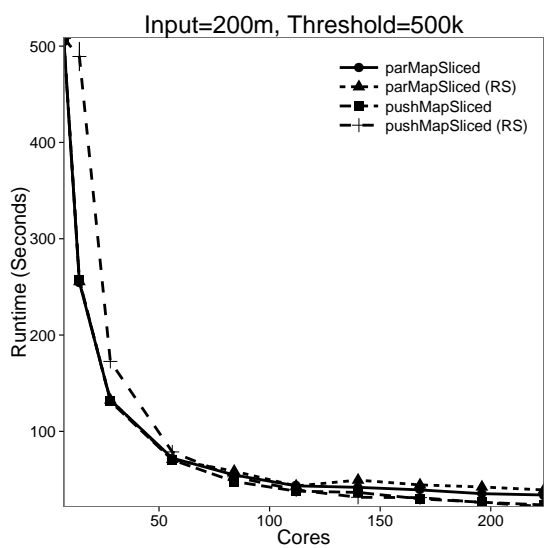

(a) Beowulf Runtime

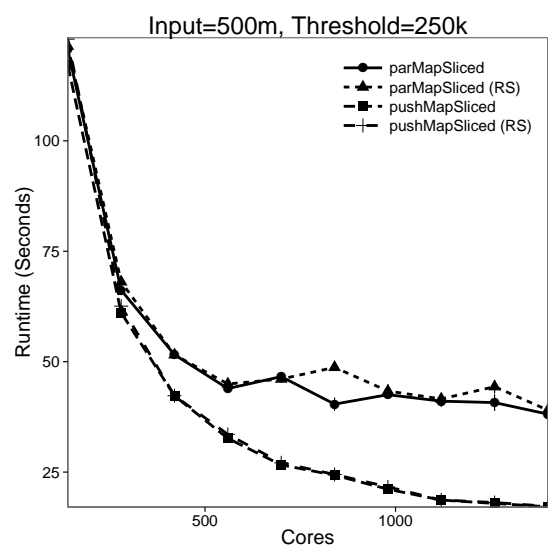

(c) HECToR Runtime

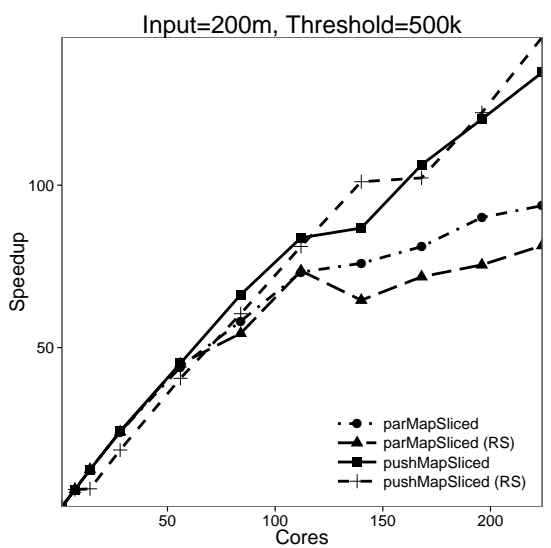

(b) Beowulf Speedup

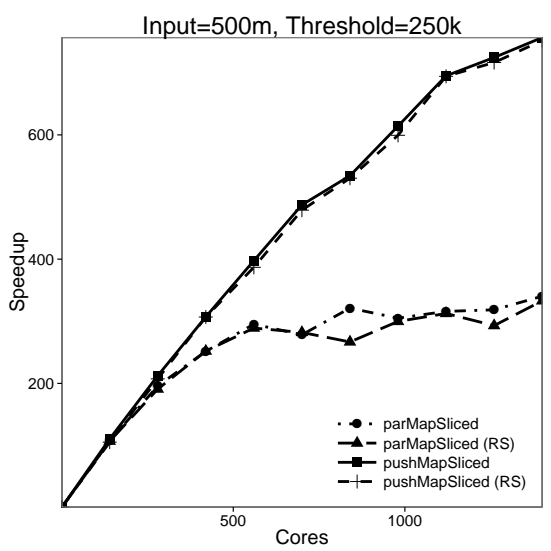

(d) HECToR Speedup

Fig. 13: Summatory Liouville on Beowulf \& HECToR

e.g. a speedup of 752 on 1400 cores, far lower than for regular HPC parallelism, but entirely acceptable for irregular symbolic computation. The exception is N Queens, which doesn't scale beyond 8 nodes, and we attribute this to a high communication to computation ratio.

\subsection{Performance With Recovery}

The HdpH-RS scheduler is designed to survive simultaneous failures. Recovery from node failure is distributed and asynchronous, the failure of a node will eventually be detected by all healthy nodes. A node replicates tasks corresponding to the supervised futures it hosts, in accordance with the DEADNODE message handler in Algorithm 1 from Section 4.4

The experiment inputs in this section are set so that failure-free runtime is a little more than 60 s on 20 Beowulf nodes. Five nodes are scheduled to die from 10 s to $60 \mathrm{~s}$, at $10 \mathrm{~s}$ intervals, from the start of execution. The runtimes are compared to failure-free runs using the equivalent non-fault tolerant skeleton to assess recovery times. Two benchmarks are 


\begin{tabular}{lccccccccc}
\hline & \multicolumn{3}{c}{ 244 Beowulf cores } & \multicolumn{4}{c}{ 1400 HECToR cores } \\
& \multicolumn{3}{c}{ lazy } & \multicolumn{2}{c}{ eager } & \multicolumn{3}{c}{ lazy } & \multicolumn{2}{c}{ eager } \\
& N-FT & FT & N-FT & FT & N-FT & FT & N-FT & FT \\
\hline N-Queens & 4 & 5 & 1 & 2 & - & - & - & - \\
Sum Euler & 85 & 73 & 113 & 114 & - & - & - & - \\
Summatory Liouville & 94 & 81 & 135 & 146 & 340 & 333 & 757 & 752 \\
Mandelbrot & 60 & 57 & 57 & 57 & 50 & 56 & 91 & 93 \\
\hline
\end{tabular}

Table 4: HdpH-RS Speedup Summary (Strong Scaling)

used to measure recovery overheads, Summatory Liouville for task parallelism and Mandelbrot for divide-and-conquer parallelism. All Summatory Lioville tasks are generated by the root node, and tasks are not recursively decomposed. Executing Mandelbrot generates divide-and-conquer supervision pattern, i.e. generates futures across multiple nodes. The recovery costs of simultaneous failure are in Figure 14 . The annotated horizontal dashed lines show mean of 5 runtimes in the absence of failure.

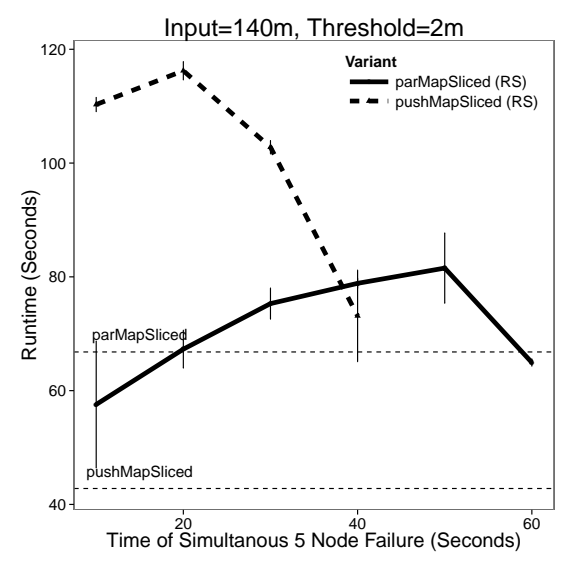

(a) Summatory Liouville

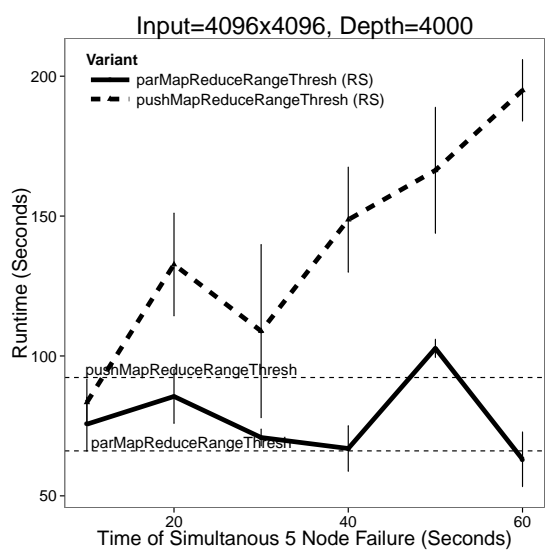

(b) Mandelbrot

Fig. 14: Recovery Costs of Simultaneous Failure of Five Beowulf Nodes

Summatory Liouville The recovery costs of Summatory Liouville are shown in Figure 14a. 70 tasks are generated. The mean runtime with parMapSliced is $66.8 \mathrm{~s}$, and $42.8 \mathrm{~s}$ with pushMapSliced. When eager scheduling is used, the recovery overheads are more substantial early on i.e. at $10 \mathrm{~s}, 20 \mathrm{~s}$ and $30 \mathrm{~s}$. These overheads compared with fault-free execution with pushMapSliced with RS are $158 \%, 172 \%$ and $140 \%$ respectively. As more tasks are evaluated, the recovery costs reduce. There are no measurements taken at $50 \mathrm{~s}$ and $60 \mathrm{~s}$ as the mean failure-free runtime with pushMapSliced is $43 \mathrm{~s}$, so killing the nodes at these times have no effect. Using lazy work stealing with parMapSliced with RS, the mean runtime is shorter than failure-free execution with parMapSliced by $14 \%$, likely 
due to the small tasks resulting from the inputs to the program - executing them on the root node is cheaper than transmitting them for remote execution. As the delay until failure is increased to 20,30, 40 and 50s, the recovery overheads are $2 \%, 13 \%, 18 \%$ and $22 \%$. Most futures on the root node are full towards the 60 second runtime, so few or no sparks need replicating which accounts for the runtimes with failure at 60 s matching runtimes with no failure.

Mandelbrot The recovery costs for Mandelbrot are shown in Figure 14b The inputs for Mandelbrot are $X=4096, Y=4096$, threshold $=4$ and depth $=4000.1023$ tasks are generated. The mean runtime with parMapReduce is 66s, and 92s with pushMapReduce. The recovery overheads for the lazy parMapReduce skeleton with RS are low, even as the number of generated supervised futures increases. The recovery overheads for the eager pushMapReduceRangeThresh skeleton with RS increases as more threads are replicated needlessly, e.g. memoization (Michie, 1968) is not employed. When the 5 node failure occurs at 20,30, 40, 50 and 60 s, the recovery costs increase, with recovery overheads of $44 \%, 18 \%, 59 \%, 80 \%$ and $110 \%$ respectively.

\subsection{Random Fault Injection with Chaos Monkey}

Random failure can be introduced to HdpH-RS programs, analogous to Netflix's Chaos Monkey (Hoff, 2010). This mechanism is used to ensure that HdpH-RS returns a result in chaotic and unreliable environments. The Chaos Monkey implementation for $\mathrm{HdpH}-$ RS is described in (Stewart, 2013b). A unit testing suite implemented with the HUnit test framework (Herington, 2006-2013) is used on the four HdpH-RS benchmarks running on 10 Beowulf nodes to check that results computed by HdpH-RS in the presence of random failure are correct. The test-suite passes $100 \%$ of the unit tests.

Chaos Monkey Results The results of chaos monkey unit testing is shown in Table B 1 in Appendix B It shows a list of integers indicating failures. Each value in the list indicates the time in seconds when each node failed. It also shows the sum of tasks that were replicated to recover from these failure(s). For the lazy scheduling skeletons, only sparks are generated and thus recovered. For the eager scheduling skeletons, only threads are generated and recovered.

For all benchmarks, lazy scheduling reduces recovery costs in the presence of failure. For example, the N-Queens benchmark generates 65234 tasks. There is an execution with eight node failures between $3 \mathrm{~s}$ and $48 \mathrm{~s}$, resulting in 40696 tasks being replicated and eagerly distributed. This results in a runtime of $650 \mathrm{~s}$, compared to the mean failure-free runtime with eager scheduling of $15 \mathrm{~s}$. This is probably due to multiple replications of tasks, due to losses of tasks higher in the supervision tree. In contrast, N-Queens with lazy scheduling and eight node failures results in only 8 tasks being replicated as sparks. The runtime is $52 \mathrm{~s}$, compared to a failure-free runtime with lazy scheduling of $28 \mathrm{~s}-\mathrm{a}$ much lower overhead than recovery with eager scheduling. A similar recovery pattern for lazy versus eager scheduling is shown for Sum Euler, Summatory Liouville and Mandelbrot. For example, Mandelbrot executions with 6 and 8 node failures incur 419 and 686 task 
replications (of the total 1023 tasks) respectively. Two Mandelbrot executions with 8 node failures incur 0 and 6 task replications respectively.

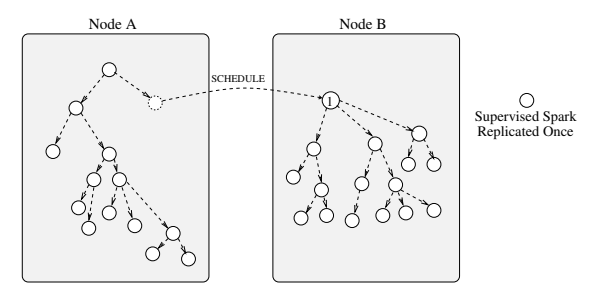

(a) Supervision with Lazy Scheduling

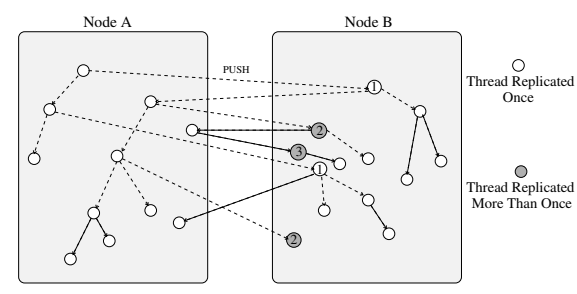

(b) Supervision with Eager Scheduling

Fig. 15: Recovering Lazy \& Eager Scheduling

Recovery Overheads with Eager Scheduling The Chaos Monkey results shows that eager scheduling incurs high levels of task replication and high runtime recovery costs. It is very likely that many threads are unnecessarily re-scheduled, as HdpH-RS does not currently cache completed task results. For the simple example of lazy scheduling shown in Figure $15 \mathrm{a}$ far fewer tasks are exposed to the risk of remote node failure as nodes only obtain a task when they are idle. The reason for the high level of rescheduling with eager skeletons is illustrated in Figure $15 \mathrm{~b}$, namely more tasks are vulnerable to node failure. Moreover, the supervision tree will be regenerated at each level, replicating children further down the task graph multiple times, even if tasks at the leaves have completed.

\section{Conclusion}

We investigate an alternative to Erlang style explicit supervision and recovery of stateful distributed actors, namely the transparent supervision and recovery of idempotent tasks. We introduce the HdpH-RS DSL which transparently adds Reliable Scheduling to HdpH. That is, the distributed fork/join-style API of HdpH carries over to HdpH-RS unchanged, including libraries of high-level skeleton abstractions like parMapSliced (Section 3.1). We provide an operational semantics for HdpH-RS, modeling task migration due to work stealing and task replication due to fault recovery (Section 3.4p. Fault recovery is semantically unobservable, i.e. does not change the result of computations provided all tasks are idempotent.

We present the fault tolerant distributed work stealing protocol of HdpH-RS in detail. Under the protocol a task's creator becomes its supervisor: tracking the task's migration due to work stealing, and replicating the task if it may have been lost due to node failure (Sections 4, 6. We use model checking to validate that the protocol honours the operational semantics (Section 5 ).

Performance evaluation shows that all benchmarks survive Chaos Monkey fault injection; and that fault tolerance overheads are consistently low in the absence of faults. HdpHRS well scales on both conventional clusters and HPC architectures. With tasks of similar sizes, eager scheduling scales better than lazy work stealing, but when failures occur the 
overheads of recovery are much lower with lazy work stealing as there are fewer tasks to recover (Section 7).

The current fault tolerance model in HdpH-RS has a number of limitations that could be addressed in future work. It would be intriguing to thoroughly explore the determinism properties of transparent fault tolerance in languages like HdpH-RS. The implementation could be improved in various ways: by integrating HdpH-RS with the the topology aware HdpH implementation (Section 6.4); by extending the communication layer to allow nodes to join a computation; and by avoiding the scalability limitation imposed by instantiating a fully connected graph of nodes during execution as in SD Erlang (Chechina et al., 2014). The current implementation re-evaluates any tasks created by a lost task, and this may be very expensive e.g. as we have seen for divide-and-conquer programs. Some form of memoization could avoid this re-evaluation. HdpH-RS could be made a more complete reliable language by providing support for reliable distributed data structures, e.g. a replicated distributed hash table or Erlang's MNesia (Mattsson et al., 1999). Such a language would support reliable big computation over reliable distributed data structures.

\section{Acknowledgments}

The work was funded by EPSRC grants HPC-GAP (EP/G05553X), AJITPar (EP/L000687/1) and Rathlin (EP/K009931/1), and EU grant RELEASE (FP7-ICT 287510). The authors thank Blair Archibald and the anonymous referees for helpful feedback.

\section{References}

Aljabri, Malak, Loidl, Hans-Wolfgang, \& Trinder, Phil W. (2014). The Design and Implementation of GUMSMP: A Multilevel Parallel Haskell Implementation. Pages 37-48 of: Proc. Implementation and Application of Functional Languages (IFL'13). New York, NY, USA: ACM.

Armstrong, Joe. (2010). Erlang. Communications of the ACM, 53(9), 68-75.

Barroso, Luiz André, Clidaras, Jimmy, \& Hölzle, Urs. (2013). The Datacenter as a Computer. 2nd edn. Morgan \& Claypool.

Boije, Jenny, \& Johansson, Luka. 2009 (December). Distributed Mandelbrot Calculations. Tech. rept. TH Royal Institute of Technology.

Borwein, Peter B., Ferguson, Ron, \& Mossinghoff, Michael J. (2008). Sign changes in Sums of the Liouville Function. Mathematics of Computation, 77(263), 1681-1694.

Cappello, Franck. (2009). Fault Tolerance in Petascale/ Exascale Systems: Current Knowledge, Challenges and Research Opportunities. IJHPCA, 23(3), 212-226.

Chandy, K. Mani, \& Lamport, Leslie. (1985). Distributed Snapshots: Determining Global States of Distributed Systems. ACM Transactions Computer Systems, 3(1), 63-75.

Chechina, Natalia, Li, Huiqing, Ghaffari, Amir, Thompson, Simon, \& Trinder, Phil. (2014). Improving Network Scalability of Erlang. Submitted to Journal of Parallel and Distributed Computing, December.

Cleary, Stephen. 2009 (May). Detection of Half-Open (Dropped) Connections. Tech. rept. Microsoft. http://blog.stephencleary.com/2009/05/detection-of-half-open-dropped.html

Cole, Murray I. (1988). Algorithmic Skeletons: A Structured Approach to the Management of Parallel Computation. Ph.D. thesis, Computer Science Department, University of Edinburgh.

Dean, Jeffrey, \& Ghemawat, Sanjay. (2008). MapReduce: Simplified Data Processing on Large Clusters. Communications of the ACM, 51(1), 107-113. 
Dinu, Florin, \& Ng, T. S. Eugene. (2011). Hadoop's Overload Tolerant Design Exacerbates Failure Detection and Recovery. 6th International Workshop on Networking Meets Databases, NETDB 2011. Athens, Greece., June.

Edinburgh Parallel Computing Center (EPCC). (2008). HECToR National UK Super Computing Resource, Edinburgh. https://www.hector.ac.uk

Elnozahy, E. N., Alvisi, Lorenzo, Wang, Yi-Min, \& Johnson, David B. (2002). A Survey of RollbackRecovery Protocols in Message-Passing Systems. Acm computing surveys, 34(3), 375-408.

Epstein, Jeff, Black, Andrew P., \& Jones, Simon L. Peyton. (2011). Towards Haskell in the Cloud. Pages 118-129 of: Proceedings of the 4th ACM SIGPLAN Symposium on Haskell, Haskell 2011, Tokyo, Japan, 22 September 2011.

Gupta, Munish. (2012). Akka essentials. Packt Publishing Ltd.

Ha, Sangtae, Rhee, Injong, \& Xu, Lisong. (2008). CUBIC: A New TCP-Friendly High-Speed TCP Variant. Operating systems review, 42(5), 64-74.

Halstead Jr., Robert H. (1985). Multilisp: A Language for Concurrent Symbolic Computation. ACM Transactions on Programming Languages and Systems, 7(4), 501-538.

Hammond, Kevin, Zain, Abdallah Al, Cooperman, Gene, Petcu, Dana, \& Trinder, Philip W. (2007). SymGrid: A Framework for Symbolic Computation on the Grid. Pages 457-466 of: 13th International Euro-Par Conference, Rennes, France, August 28-31, 2007, Proceedings.

Harris, Tim, Marlow, Simon, \& Jones, Simon L. Peyton. (2005). Haskell on a Shared-Memory Multiprocessor. Pages 49-61 of: Proceedings of the ACM SIGPLAN Workshop on Haskell, Haskell 2005, Tallinn, Estonia, September 30, 2005.

Herington, Dean. (2006-2013). Haskell library: hunit package. A unit testing framework for Haskell. http://hackage.haskell.org/package/HUnit

Hoff, Todd. 2010 (December). Netflix: Continually Test by Failing Servers with Chaos Monkey. http://highscalability.com.

Holzmann, Gerard J. (2004). The SPIN Model Checker - Primer and Reference Manual. AddisonWesley.

John, Annu, Konnov, Igor, Schmid, Ulrich, Veith, Helmut, \& Widder, Josef. (2013). Towards Modeling and Model Checking Fault-Tolerant Distributed Algorithms. Pages 209-226 of: Model Checking Software - Proceedings of 20th International Symposium, SPIN 2013, Stony Brook, NY, USA, July, 2013.

Kuper, Lindsey, Turon, Aaron, Krishnaswami, Neelakantan R., \& Newton, Ryan R. (2014). Freeze After Writing: Quasi-Deterministic Parallel Programming with LVars and Handlers. POPL 2014, San Diego, USA. ACM.

Lamport, Leslie. (1978). Time, Clocks, and the Ordering of Events in a Distributed System. Communications of the acm, 21(7), 558-565.

Litvinova, Antonina, Engelmann, Christian, \& Scott, Stephen L. 2010 (Feb. 16-18,). A Proactive Fault Tolerance Framework for High-Performance Computing. Proceedings of the 9th IASTED International Conference on Parallel and Distributed Computing and Networks (PDCN) 2010.

Loogen, Rita, Ortega-Mallén, Yolanda, \& Peña-Marí, Ricardo. (2005). Parallel functional programming in Eden. J. funct. program., 15(3), 431-475.

Maier, Patrick, \& Trinder, Phil. (2012). Implementing a High-level Distributed-Memory Parallel Haskell in Haskell. Pages 35-50 of: Implementation and Application of Functional Languages, 23rd International Symposium 2011, Lawrence, KS, USA, October 3-5, 2011. Revised Selected Papers. Lecture Notes in Computer Science, vol. 7257. Springer.

Maier, Patrick, Livesey, Daria, Loidl, Hans-Wolfgang, \& Trinder, Phil. (2014a). High-performance computer algebra: A Hecke algebra case study. Pages 415-426 of: Silva, Fernando M. A., de Castro Dutra, Inês, \& Costa, Vítor Santos (eds), Euro-par 2014 parallel processing - 20th 
international conference, porto, portugal, august 25-29, 2014. proceedings. Lecture Notes in Computer Science, vol. 8632. Springer.

Maier, Patrick, Stewart, Robert J., \& Trinder, Philip W. (2014b). Reliable Scalable Symbolic Computation: The Design of SymGridPar2. Computer Languages, Systems \& Structures, 40(1), 19-35.

Maier, Patrick, Stewart, Robert J., \& Trinder, Phil. (2014c). The HdpH DSLs for Scalable Reliable Computation. Pages 65-76 of: Proceedings of the 2014 ACM SIGPLAN Symposium on Haskell, Gothenburg, Sweden, September 4-5, 2014. ACM.

Marlow, Simon, \& Newton, Ryan. (2013). Source code for monad-par library. https://github. com/simonmar/monad-par

Marlow, Simon, Jones, Simon L. Peyton, \& Singh, Satnam. (2009). Runtime Support for Multicore Haskell. Pages 65-78 of: Icfp.

Marlow, Simon, Newton, Ryan, \& Jones, Simon L. Peyton. (2011). A Monad for Deterministic Parallelism. Pages 71-82 of: Proceedings of the 4th ACM SIGPLAN Symposium on Haskell, Haskell 2011, Tokyo, Japan, 22 September 2011.

Mattsson, Hkan, Nilsson, Hans, \& Wikstrm, Claes. (1999). Mnesia - A Distributed Robust DBMS for Telecommunications Applications. Pages 152-163 of: PADL.

Meredith, Marsha, Carrigan, Teresa, Brockman, James, Cloninger, Timothy, Privoznik, Jaroslav, \& Williams, Jeffery. (2003). Exploring Beowulf Clusters. Journal of Computing Sciences in Colleges, 18(4), 268-284.

Michie, Donald. (1968). "Memo" Functions and Machine Learning. Nature, 218(5136), 19-22.

Peyton Jones, Simon. (2002). Tackling the Awkward Squad: Monadic Input/Output, Concurrency, Exceptions, and Foreign-Language Calls in Haskell. Pages 47-96 of: Engineering Theories of Software Construction.

Pnueli, Amir. (1977). The Temporal Logic of Programs. Pages 46-57 of: 18th Annual Symposium on Foundations of Computer Science, Providence, Rhode Island, USA, 31 October - 1 November 1977. IEEE Computer Society.

Postel, J. 1980 (August). User Datagram Protocol. RFC 768 Standard. http://www.ietf .org/ rfc/rfc768.txt.

Prior, Arthur N. (1957). Time and Modality. Oxford University Press.

Ramalingam, Ganesan, \& Vaswani, Kapil. (2013). Fault Tolerance via Idempotence. Pages 249-262 of: Popl.

Rivin, Igor, Vardi, Ilan, \& Zimmerman, Paul. (1994). The N-Queens Problem. The American Mathematical Monthly, 101(7), pp. 629-639.

Scholz, Sven-Bodo. (2003). Single Assignment C: Efficient Support for High-level Array Operations in a Functional Setting. J. Funct. Program., 13(6), 1005-1059.

Schroeder, Bianca, \& Gibson, Garth A. (2007). Understanding Failures in Petascale Computers. Journal of Physics: Conference Series, 78, 012022 (11pp). http://stacks.iop.org/ $1742-6596 / 78 / 012022$

Scott, J., \& Kazman, R. (2009). Realizing and Refining Architectural Tactics: Availability. Technical report. Carnegie Mellon University, Software Engineering Institute.

Stewart, Robert. 2013a (December). Promela Abstraction of the HdpH-RS Scheduler. https://github.com/robstewart57/phd-thesis/blob/master/spin_model/ hdph_scheduler.pml

Stewart, Robert. 2013b (November). Reliable Massively Parallel Symbolic Computing: Fault Tolerance for a Distributed Haskell. Ph.D. thesis, Mathematical and Computer Sciences, HeriotWatt University, Edinburgh, Scotland.

Stewart, Robert, \& Maier, Patrick. (2013). HdpH-RS source code. https://github.com/ robstewart57/hdph-rs 
Stewart, Robert, Maier, Patrick, \& Trinder, Phil. 2015 (June). Open access dataset for "Transparent Fault Tolerance for Scalable Functional Computation". http://dx.doi.org/10.5525/gla. researchdata.189

Trinder, Philip W., Hammond, Kevin, Jr., James S. Mattson, Partridge, A. S., \& Jones, Simon L. Peyton. (1996). GUM: A Portable Parallel Implementation of Haskell. Pages 79-88 of: Proc. ACM Programming Language Design and Implementation (PLDI'96), Philadephia, Pennsylvania, May.

White, Tom. (2012). Hadoop - The Definitive Guide: Storage and Analysis at Internet Scale (3. ed., revised and updated). O'Reilly.

Xu, Chengzhong, \& Lau, Francis C. (1997). Load Balancing in Parallel Computers: Theory and Practice. Norwell, MA, USA: Kluwer Academic Publishers. 


\section{A Promela Abstraction of the Verified HdpH-RS Scheduler}

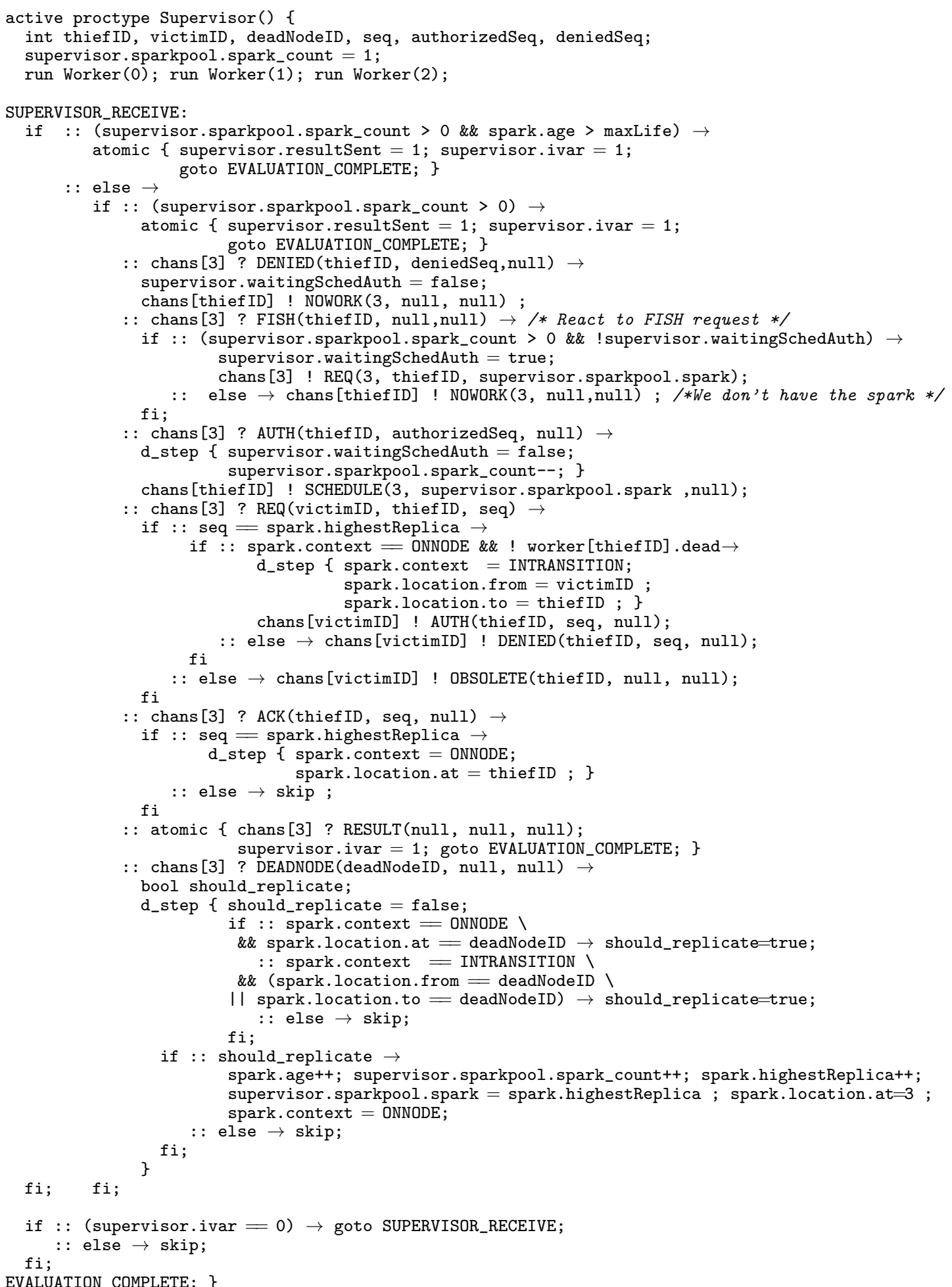

Fig. A 1: Repetitive Control Flow Options for Supervisor 
proctype Worker (int me) \{

int thiefID, victimID, deadNodeID, seq, authorisedSeq, deniedSeq;

WORKER_RECEIVE:

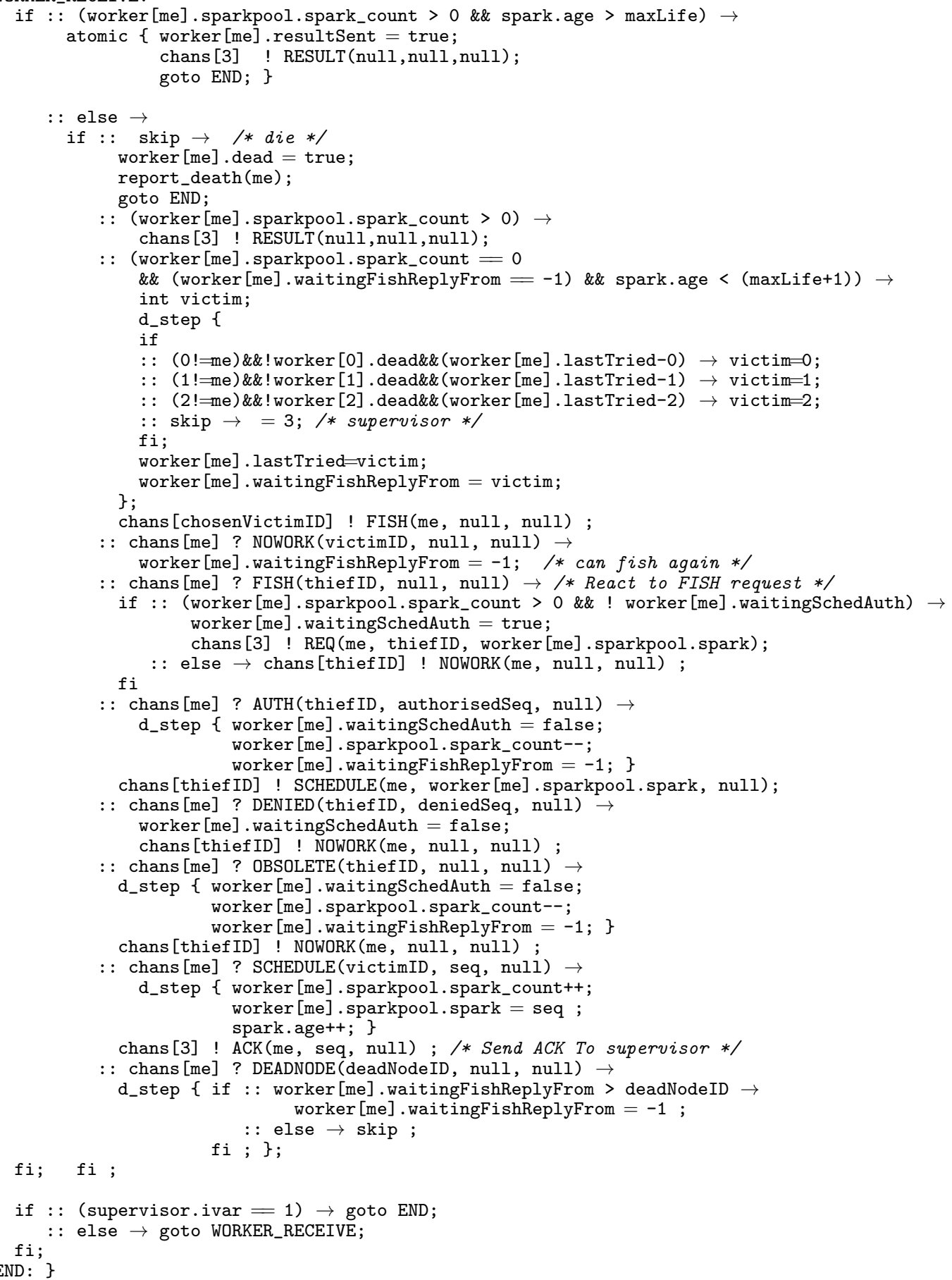

Fig. A 2: Repetitive Control Flow Options for a Worker 


\section{B HdpH-RS Chaos Monkey Results}

\begin{tabular}{|c|c|c|c|c|c|c|}
\hline \multirow{2}{*}{ Benchmark } & \multirow{2}{*}{ Skeleton } & \multirow{2}{*}{$\begin{array}{l}\text { Failed Nodes } \\
\text { (seconds) }\end{array}$} & \multicolumn{2}{|c|}{ Recovery } & \multirow{2}{*}{$\begin{array}{l}\text { Runtime } \\
\text { (seconds) }\end{array}$} & \multirow{2}{*}{ Unit Test } \\
\hline & & & & Threads & & \\
\hline \multirow{11}{*}{$\begin{array}{l}\text { Sum Euler } \\
\text { lower }=0 \\
\text { upper }=100000 \\
\text { chunk }=100 \\
\text { tasks }=1001 \\
X=3039650754\end{array}$} & parMapChunked & - & & & 126.1 & pass \\
\hline & & {$[6,30,39,49,50]$} & 10 & & 181.1 & pass \\
\hline & & {$[5,11,18,27,28,33,44,60]$} & 16 & & 410.2 & pass \\
\hline & parMapChunked (RS) & {$[31,36,49]$} & 6 & & 139.7 & pass \\
\hline & & $\begin{array}{c}{[37,48,59]} \\
{[1,17,24,27,43,44,47,48,48]}\end{array}$ & $\begin{array}{c}6 \\
17\end{array}$ & & $\begin{array}{l}139.5 \\
768.2\end{array}$ & $\begin{array}{l}\text { pass } \\
\text { pass }\end{array}$ \\
\hline & pushMapChunked & ( & & & 131.6 & pass \\
\hline & & {$[4,34,36,37,48,49,58]$} & & & & \\
\hline & & {$[2,6,11,15,17,24,32,37,41]$} & & 915 & 1179.7 & $\begin{array}{l}\text { pass } \\
\text { pass }\end{array}$ \\
\hline & pushMapChunked (RS) & {$[2,37,39,45,49]$} & & 481 & 564.0 & pass \\
\hline & & {$[4,7,23,32,34,46,54,60]$} & & 760 & 978.1 & pass \\
\hline & & {$[35,38,41,43,46,51]$} & & 548 & 634.3 & pass \\
\hline \multirow{11}{*}{$\begin{array}{l}\text { Summatory } \\
\text { Liouville } \\
\lambda=50000000 \\
\text { chunk }=100000 \\
\text { tasks }=500 \\
X=-7608\end{array}$} & parMapSliced & - & & & 56.6 & pass \\
\hline & & {$[32,37,44,46,48,50,52,57]$} & 16 & & 85.1 & pass \\
\hline & norManCliced (RC) & {$[18,27,41]$} & 6 & & 61.6 & pass \\
\hline & parmapsılced (KS) & {$[19,30,39,41,54,59,59]$} & 14 & & 76.2 & pass \\
\hline & & $\begin{array}{c}{[8,11]} \\
{[8,9,24,28,32,34,40,57]}\end{array}$ & $\begin{array}{c}4 \\
16\end{array}$ & & $\begin{array}{c}62.8 \\
132.7\end{array}$ & pass \\
\hline & & {$\left[\begin{array}{ll}1 \\
\end{array}\right.$} & & & & \\
\hline & pushMapsliced & - & & & 58.3 & pass \\
\hline & & {$[3,8,8,12,22,26,26,29,55]$} & & 268 & 287.1 & pass \\
\hline & pushMapSliced (RS) & $\begin{array}{c}{[1]} \\
{[10,59]}\end{array}$ & & $\begin{array}{l}53 \\
41\end{array}$ & $\begin{array}{l}63.3 \\
68.5\end{array}$ & pass \\
\hline & & {$[13,15,18,51]$} & & $\begin{array}{l}41 \\
106\end{array}$ & 125.0 & pass \\
\hline & & {$[13,24,42,51]$} & & 80 & 105.9 & pass \\
\hline \multirow{12}{*}{$\begin{array}{l}\text { Queens } \\
14 \times 14 \text { board } \\
\text { threshold }=5 \\
\text { tasks }=65234 \\
X=365596\end{array}$} & parDnC & - & & & 28.1 & pass \\
\hline & & {$[3,8,9,10,17,45,49,51,57]$} & 8 & & 52.1 & pass \\
\hline & & {$[1,30,32,33,48,50]$} & 5 & & 49.4 & pass \\
\hline & parDnC (RS) & {$[8,15]$} & 2 & & 53.3 & pass \\
\hline & & {$[20,40,56]$} & 2 & & $\begin{array}{l}49.9 \\
52.8\end{array}$ & pass \\
\hline & & [] & & & 52.8 & pass \\
\hline & pushDnC & - & & & 15.4 & pass \\
\hline & & {$[14,33]$} & & 5095 & 57.1 & pass \\
\hline & & {$[3,15,15,23,24,28,32,48]$} & & 40696 & 649.5 & pass \\
\hline & pushDnC (RS) & {$[5,8,26,41,42,42,59]$} & & 36305 & 354.9 & pass \\
\hline & & {$[0,5,8,10,14,28,31,51,54]$} & & 32629 & 276.9 & pass \\
\hline & & {$[31,31,58,60]$} & & 113 & 47.8 & pass \\
\hline \multirow{11}{*}{$\begin{array}{l}\text { Mandelbrot } \\
x=4048 \\
y=4048 \\
\text { depth }=256 \\
\text { threshold }=4 \\
\text { tasks }=1023 \\
X=449545051\end{array}$} & parMapReduce & - & & & 23.2 & pass \\
\hline & & {$[28,30,36,44,49,54,56,56]$} & 0 & & 29.1 & pass \\
\hline & & [] [] & 0 & & 27.8 & pass \\
\hline & parMapReduce (RS) & {$[7,24,25,25,44,53,54,59]$} & 6 & & 32.6 & pass \\
\hline & & $\begin{array}{c}{[17,30]} \\
{[0,14]} \\
\end{array}$ & $\begin{array}{l}0 \\
2\end{array}$ & & $\begin{array}{l}55.4 \\
33.7\end{array}$ & $\begin{array}{l}\text { pass } \\
\text { pass }\end{array}$ \\
\hline & pushMapReduce & - & & & 366.3 & pass \\
\hline & & {$[9,24,34,34,52,59]$} & & 419 & 205.3 & \\
\hline & & {$[7,8,11,22,32,35,44,46]$} & & 686 & 395.9 & pass \\
\hline & pushMapReduce (RS) & {$[27,49]$} & & 2 & 371.8 & pass \\
\hline & & [9 33505052531 & & $\begin{array}{c}0 \\
84\end{array}$ & 380.4 & pass \\
\hline & & {$[9,33,50,50,52,53]$} & & 84 & 216.1 & pass \\
\hline
\end{tabular}

Table B 1: Fault Tolerance Unit Testing: Chaos Monkey Runtimes 